\title{
LA -NUREG-6570-MS
}

Informal Report

NRC-8

\section{LEAF:}

A Computer Program to Calculate Fission Product Release from a Reactor Containment Building for Arbitrary Radioactive Decay Chains

Issued: November 1976 


\section{DISCLAIMER}

This report was prepared as an account of work sponsored by an agency of the United States Government. Neither the United States Government nor any agency Thereof, nor any of their employees, makes any warranty, express or implied, or assumes any legal liability or responsibility for the accuracy, completeness, or usefulness of any information, apparatus, product, or process disclosed, or represents that its use would not infringe privately owned rights. Reference herein to any specific commercial product, process, or service by trade name, trademark, manufacturer, or otherwise does not necessarily constitute or imply its endorsement, recommendation, or favoring by the United States Government or any agency thereof. The views and opinions of authors expressed herein do not necessarily state or reflect those of the United States Government or any agency thereof. 


\section{DISCLAIMER}

Portions of this document may be illegible in electronic image products. Images are produced from the best available original document. 
This work was supported by the US Nuclear Regulatory Commission, Office of Nuclear Regulatory Research, Division of Reactor Safety Research.

\author{
Printed in the United States of America. Available from \\ National Technical Information Service \\ U.S. Department of Commerce \\ 5285 Port Royal Road \\ Springfield, VA 22161 \\ Price: Printed Copy $\$ 4.00$ Microfiche $\$ 3.00$
}

\title{
NOTICE
}

This report was prepared as an account of work sponsored by the United States Government. Neither the United States nor the United States Nuclear Regulatory Commission, nor any of their employees, nor any of their contractors, subcontractors, or ' their employees, makes any warranty, express or implied, or assumes any legal liability or responsibility for the accuracy, completeness or usefulness of any information, apparatus, product or process disclosed, or represents that its use would not infringe privately owned rights. 


\section{LEAF:}

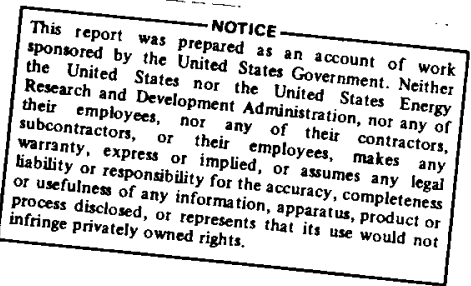

\section{A Computer Program to Calculate Fission Product Release from a Reactor Containment Building for Arbitrary Radioactive Decay Chains}

by

Clarence E. Lee

Courtney E. Apperson, Jr. John E. Foley

Manuscript completed: October 1976

Issued: November 1976 


\section{LEAF: A COMPUTER PROGRAM TO CALCULATE FISSION PRODUCT RELEASE}

FROM A REACTOR CONTAINMENT BUILDING FOR ARBITRARY RADIOACTIVE DECAY CHAINS

by

Clarence E. Lee, Courtney E. Apperson, Jr., and John E. Foley

\section{ABSTRACT}

This report describes an analytic containment building model that is used for calculating the leakage into the environment of each isotope of an arbitrary radioactive decay chain. The model accounts for the source, the buildup, the decay, the cleanup, and the leakage of isotopes that are gas-borne inside the containment building.

\section{INTRODUCTION}

The sources of the radioactive materials that are needed for the calculation of the consequences of postulated reactor accidents are obtained from estimates of the leakage of fission products from the reactor containment building. These estimates are obtained from a mathematical model of the reactor containment build- ing that accounts for the source, the decay, the cleanup, and the leakage of each radionuclide in the building. A containment building model, which assumes that the gas in the building consists of a single, well mixed volume, is described in the Reactor Safety Study. ${ }^{1}$ We have used a similar mode ${ }^{2,3}$ to estimate the timedependent release of ${ }^{131}$ I from the containment building of a High-Temperature Gas-Cooled Reactor (HTGR) during the Loss of Forced Circulation (LOFC) accident. The containment building model of References 2 and 3 is useful only for single isotopes because the radioactive decay chains are not included.

In this report we describe an analytic containment building model that is used for calculating the leakage into the environment of each isotope of an 
arbitrary radioactive decay chain. The model accounts for the source, the buildup, the decay, the cleanup, and the leakage of the isotopes that are gas-borne inside the containment building. In the model, the source of an isotope inside the containment building (which is the result of leakage from the reactor vessel), its removal rate by the containment cleanup system, and its leakage from the containment building are a11 assumed to be constant during short time intervals. We assume, as is done in Ref. 1, that the gas inside the containment building is well mixed and all in one compartment. Natural deposition of gas-borne isotopes onto surfaces internal to the containment building was not included in the model.

Even though we use this containment building model to estimate the timedependent release of fission products to the environment for postulated HTGR accidents, the model is quite general and can be used for other types of reactors.

\section{LEAF MODEL EQUATIONS}

We consider a system shown in $\mathrm{Fig}$. 1, composed of a reactor vessel emitting a source of radioactive materials $\underline{S}$, surrounded by a containment building which leaks at a rate $L\left(s^{-1}\right)$. Inside the containment building there is a cleanup system filter having a cleanup rate $V\left(s^{-1}\right)$. Let $\underline{N}$ be the amount of an isotope of a chain in the containment building, and $\underline{F}$ the total amount of the isotope absorbed on the filter. $\underline{N}, \underline{F}$, and $\underline{S}$ are vectors, the elements of which are the values of the individual species in the chain.

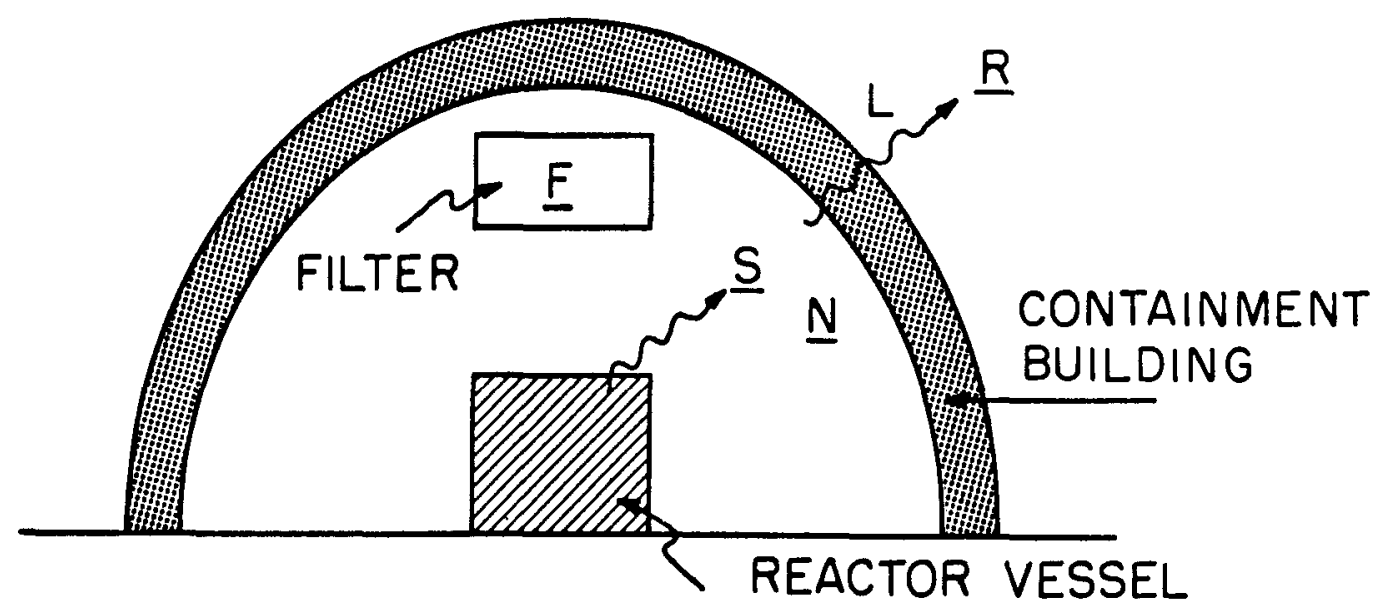

Fig. 1. LEAF containment building model. 
Denote $\bar{\lambda}$ the decay chain matrix, and $L$ and $V$ the diagonal leak and filter cleanup rate matrices. The negative off-diagonal elements of $\bar{\lambda}$ include the branching ratio factors; the diagonal elements of $\bar{\lambda}$ are positive. Noble gases will not be filtered by the cleanup system. This fact is represented by a matrix $\bar{\delta}$ of the form

$$
\bar{\delta}_{i j}=\mu_{i}\left(1-\delta_{i j}\right),
$$

where

$$
\mu_{i}=\left\{\begin{array}{l}
1 \text { if the } i^{\text {th }} \text { isotope is a noble gas } \\
0 \text { otherwise }
\end{array}\right.
$$

and $\delta_{i j}$ is the Kronecker de 7 ta, defined by

$$
\delta_{i j}= \begin{cases}1 & \text { if } i=j \\ 0 & \text { if } i \neq j\end{cases}
$$

Defining the matrices $\lambda, \lambda^{*}$, and $\Lambda$ by

$$
\begin{aligned}
& \lambda=\bar{\lambda}-\lambda^{*}, \\
& \lambda^{\star}=\bar{\delta} \bar{\lambda}, \\
& \Lambda=\bar{\lambda}+V+L,
\end{aligned}
$$

where denotes the Cartesian product, then the LEAF model equations are written as

$$
\begin{aligned}
& \frac{d \underline{N}}{d t}=-\Lambda \underline{N}-\lambda^{*} \underline{F}+\underline{S}, \\
& \frac{d F}{d t}=V \underline{N}-\lambda \underline{F} .
\end{aligned}
$$

If we define new vectors $\underline{X}$ and $\underline{s}$ by

$$
\underline{X}=\left(\frac{N}{F}\right), \underline{s}=\left(\frac{S}{0}\right) \text {, }
$$

and a supermatrix $A$ by

$$
A=\left(\begin{array}{cc}
-\Lambda & -\lambda^{\star} \\
V & -\lambda
\end{array}\right) \text {, }
$$

then Eq. (5) may be rewritten as

$$
\frac{d \underline{X}}{d t}=A \underline{x}+\underline{s} \text {. }
$$

We assume that the matrix $A$ and the vector $\underline{s}$ are constant over the time interval $(0, \tau)$. 
We are interested in calculating the integrated release to the environment by leakage from the containment building. This is given by

$$
\begin{aligned}
\underline{R}(\tau) & =\int_{0}^{\tau} d t^{\prime} L\left(t^{\prime}\right) \underline{N}\left(t^{\prime}\right) \\
& =\bar{L} \int_{0}^{\tau} d t^{\prime} \underline{N}\left(t^{\prime}\right),
\end{aligned}
$$

where $\bar{L}$ is the average leakage in the time interval $\tau$, defined by

$$
\begin{aligned}
& \bar{L}=\frac{1}{2}(L(0)+L(\tau)) . \\
& \text { If we define } \\
& \underline{Y}(\tau)=\left(\begin{array}{c}
\underline{R}(\tau) \\
0
\end{array}\right), B=\left(\begin{array}{ll}
L & 0 \\
L & 0
\end{array}\right),
\end{aligned}
$$

then Eq. (9) becomes

$$
\underline{Y}(\tau)=B \int_{0}^{\tau} d s \underline{X}(s) \text {. }
$$

As we shall prove in detail below, if the matrix $A$ is constant in the time interval $(0, \tau)$, the solutions to Eqs. (8) and (12) are given by

$$
\underline{x}(\tau)=\underline{x}(0)+\tau D(A \tau) \quad[A \underline{x}(0)+\underline{s}] \text {, }
$$

and

$$
\underline{Y}(\tau)=B\left[\tau D(A \tau) \underline{X}(0)+\tau^{2} Z(A \tau) \underline{s}\right],
$$

where the matrix operators $D(C)$ and $Z(C)$ for $C=A \tau$ are evaluable using the methods of Ref. 4 .

III. ANALYTIC SOLUTIONS TO THE LEAF MODEL EQUATIONS

Because the matrix $A$ is constant in the time interval $(0, \tau)$, then following the Volterra method of the multiplicative integral, ${ }^{4,5}$ we may construct the matricant

$$
\Omega_{0}^{\tau}(A)=\exp \left[\int_{0}^{\tau} A(s) d s\right]=\exp (A \tau) .
$$

The solution to Eq. (8) is given by

$$
\underline{x}(\tau)=\Omega_{0}^{\tau}(A) \underline{x}(0)+\int_{0}^{\tau} d t^{\prime} K\left(\tau, t^{\prime}\right) \underline{s}\left(t^{\prime}\right),
$$

where

$$
R\left(\tau, t^{\prime}\right) \equiv \Omega_{0}^{\tau}(A)\left[\Omega_{0}^{t^{\prime}}(A)\right]-1 \text {. }
$$

As is readily proved, ${ }^{4}$ both the matrix $A$ and $e^{A \tau}$ are non-negative.

Substituting Eq. (15) into Eqs. (16) and (17) gives

$$
\underline{x}(\tau)=e^{A \tau} \underline{x}(0)+e^{A \tau} \int_{0}^{\tau} d t^{\prime} e^{-A t^{\prime}} \underline{s}\left(t^{\prime}\right) \text {. }
$$


Assuming that $\underline{s}\left(t^{\prime}\right)=\underline{s}$ is constant over the interval $(0, \tau)$, Eq. (18) becomes

$$
\underline{x}(\tau)=e^{A \tau} \underline{x}(0)+A^{-1}\left(e^{A \tau}-I\right) \underline{s} .
$$

Defining the matrix operator $D(C)$ by 4

$$
D(C)=C^{-1}\left(e^{C}-I\right)
$$

or

$$
\tau D(A \tau)=A^{-1}\left(e^{A \tau}-I\right),
$$

Eq. (19) becomes

$$
\begin{aligned}
\underline{X}(\tau) & =\underline{X}(0)+\tau A D(A \tau) \underline{x}(0)+\tau D(A \tau) \underline{s} \\
& =\underline{x}(0)+\tau D(A \tau)[\underline{A} \underline{x}(0)+\underline{s}],
\end{aligned}
$$

which is Eq. (13).

In order to derive Eq. (14) we integrate Eq. (12) to obtain

$$
\begin{aligned}
\underline{Y}(\tau) & =B \quad\left\{A^{-1}\left(e^{A \tau}-I\right) \underline{x}(0)+\left[-A^{-1} \tau-A^{-1} A^{-1}\left(e^{A \tau}-I\right)\right] \underline{s}\right\} \\
& =B \quad\left\{\tau D(A \tau) \underline{x}(0)+\tau^{2}\left[-C^{-1}+C^{-1} D(C)\right] \underline{s}\right\} \\
& =B \quad\left\{\tau D(A \tau) \underline{x}(0)+\tau^{2} Z(A \tau) \underline{s}\right\},
\end{aligned}
$$

where we have defined

$$
C Z(C)=D(C)-I
$$

for $C=A \tau$ and used Eq. (21). The last line of Eq. (23) is just Eq. (14).

Note that the matrix operators $D(C)$ and $Z(C)$ defined by

$$
\begin{aligned}
& D(C)=C^{-1}\left(e^{C}-I\right)=\sum_{n=0}^{\infty} \frac{C^{n}}{(n+1) !} \text { and } \\
& Z(C)=C^{-1}(D(C)-I)=\sum_{n=0}^{\infty} \frac{C^{n}}{(n+2) !}
\end{aligned}
$$

exist even if $C=A \tau$ is singular.* Although the eigenvalues of $e^{C}$ are bounded by unity, and the eigenvalues of $C$ are bounded, but not necessarily by unity, the direct evaluation of $D(C)$ and $Z(C)$ would prove difficult computationally if Eqs. (25) and (26) are used. We can scale the matrix $C$ so that the eigenvalues are bounded by unity. Define

$$
H=2^{-p} C,
$$

where $p$ is determined by

$$
\begin{gathered}
\|H\|<\frac{1}{2} \\
\operatorname{or} 4,6>\ln \left(\sum_{i j}^{\prime}\left|C_{i j}\right|^{2}\right) /(2 \text { ln } 2) .
\end{gathered}
$$

*For example, a chain involving a stable isotope will lead to a matrix $C$ that is singular. 
We approximate the $D(H)$ and $Z(H)$ matrix operators by a finite number of terms $M$ using Eqs.(25) and (26).

$$
\begin{aligned}
D^{M}(H) & \approx \sum_{n=0}^{M} \frac{H^{n}}{(n+1) !} \\
Z^{M}(H) & \approx \sum_{n=0}^{M} \frac{H^{n}}{(n+2) !}
\end{aligned}
$$

$M$ is determined ${ }^{4}$ such that the excluded terms have an error less than some $\varepsilon$, or

$$
\frac{(|| H||)^{M+1}}{(M+2) !}<\frac{1}{2^{M+1}(M+2) !}<\varepsilon .
$$

Knowing $D(H)$ and $Z(H)$, we may recur upwards by powers of 2 in $H$ to find $D(C)$ and $Z(C)$ where $C=2 p_{H}$, using the recursion relations

$$
\begin{aligned}
& D\left(2^{p+1}+1\right)=D\left(2^{p_{H}}\right)\left[I+\frac{1}{2}\left(2^{p_{H}}\right) D\left(2^{p_{H}}\right)\right] \text { and } \\
& Z\left(2^{p+1}+1_{H}=\frac{1}{2} Z\left(2^{p_{H}}\right)+\frac{1}{4}\left[D\left(2^{p_{H}}\right)\right]^{2} .\right.
\end{aligned}
$$

These recursion relationships are proved in Appendix $A$.

Using the above equations, we wrote and debugged a computer program called LEAF. The LEAF program listing is given in Appendix B.

We next discuss the program logic of LEAF, the input structure, and then we examine some of the comparisons that were made for the validation of the LEAF program.

\section{LEAF PROGRAM LOGIC}

The LEAF program consists of a driver routine which controls the program flow (LEAF); nine primary subroutines (INPA, INPC, MAKEA, SOLVER, FSOLVE, PREP, PAPER, TERM, and PRMAT), which perform input/output tasks; and five secondary subroutines (SCALAR, MULTI, EQUAL, MVMUL, VADD), which are called by the primary subroutines to evaluate matrix and vector operations in double precision.

The dimensions of arrays are set by a parameter statement. The meaning and the current values of the four parameters in LEAF are

NNT $=10:$ Maximum number of nuclides allowed a problem,

NN $=2 \star N N T:$ Twice NNT,

NIT $=25 \quad$ : Maximum number of time intervals plus one, and

NBR $=10$ : Maximum number of branching ratios allowed. 
These parameters can be increased as long as NN $=2 * N N T$. No use is made of Large Core Memory in this version of the code. The code runs using both CROS-CDC -7600 BATCH mode and NOS-CDC-6600 time-sharing terminal mode.

The nine primary subroutines are discussed in the order in which they are called by the driver routine LEAF.

A. INPA

The subroutine INPA reads and prints the basic nuclear data used in constructing the decay chain matrices. The input is stored so that it may be recalled in subsequent subroutines. The printing of this data is controlled by the value of NSKIP. If NSKIP is greater than zero, the input read by INPA is not printed. B. INPC

The subroutine INPC reads and prints the time-dependent data, the initial concentrations, and the time-dependent source data. The initial concentrations and time-dependent sources are input in atoms and atoms/s if IAC $=0$. If IAC $>0$, the input is in $\mathrm{Cj}$ and $\mathrm{Cj} / \mathrm{s}$ for radioactive isotopes $(\lambda \neq 0)$, and in $\mathrm{g}$ and $\mathrm{g} / \mathrm{s}$ for stable isotopes $(\lambda=0)$. As in INPA, the printing of the input is controlled by the value of NSKIP.

At this point all of the required inputs have been read and stored.

C. MAKEA

The subroutine MAKEA constructs the main solution matrix $A$ defined in Eq. (7). The size of the A matrix is NN by NN. The upper half of the A matrix models the behavior of the nuclides in the containment building. The lower half of the A matrix models the behavior in the containment building filter system.

The subroutine MAKEA also constructs the matrix B, Eq. (11). B is called $\mathrm{BL}$ in MAKEA, and premultiplies the integrated containment concentration vector to calculate the integrated release to the environment.

D. SOLVER

The subroutine SOLVER uses the matrix $A$ to calculate three matrix operators: $D(A \tau), I+A \tau D(A \tau)$, and $Z(A \tau)$ as used in Eqs. (13) and (14). The value of $p$ is determined using Eq. (29) to insure that ||$H||<\frac{1}{2}$, where $H=2^{-P} C$ and $C=A \tau$. Then the power series representations for $D(H)$ and $Z(H)$ are evaluated, Eqs. (30) and (31). Finally, the recursion relations, Eqs. (33) and (34), are used to determine the $D(C), I+C D(C)$ and $Z(C)$ matrix operators needed by FSOLVE to establish the solution for a given time interval. 


\section{E. FSOLVE}

The subroutine FSOLVE calculates the concentrations of the nuclides in the containment building and filter as well as the integrated release to the environment for the specified time interval, Eqs. (13) and (14), using the matrix operators determined in SOLVER.

The system of subroutines (MAKEA, SOLVER, and FSOLVE) is repeatedly evaluated for each time interval as specified by the input read by subroutine INPC. F. PREP

Upon completion of the calculation of all the time intervals, the subroutine PREP is used to prepare the results for final output display. This subroutine converts the calculated results in atoms into curies for radioactive nuclides and into grams for stable nuclides.

G. PAPER, TERM

The results of the LEAF calculations are printed by either the subroutine PAPER or TERM. The subroutine PAPER provides a detailed and labeled presentation of the results for each time interval in atoms and in curies or grams. The subroutine TERM produces an abbreviated output for each time interval in atoms. This routine is intended for use when the output is to be displayed on an interactive terminal and is chosen when NSKIP is greater than zero.

H. PRMAT

The subroutine PRMAT prints the matrix $A$ for each time interval if the variable MATRIX is greater than zero. The A matrix is printed by quadrant in the trigonometric convention

$$
A=\left(\begin{array}{cc}
-\Lambda & -\lambda^{\star} \\
V & -\lambda
\end{array}\right),
$$

where quadrant $1=-\lambda^{\star}$, quadrant $2=-\Lambda$, quadrant $3=V$, quadrant $4=-\lambda$, as defined by Eqs. (1) through (4).

Finally, there are five secondary subroutines in LEAF which perform matrix and vector operations in double precision. These routines and their functions are as follows.

1. SCALAR: Multiplies a scalar times a matrix.

2. MULTI : Multiplies two matrices.

3. EQUAL : Sets one matrix equal to another.

4. MVMUL : Multiplies a matrix times a vector.

5. VADD : Adds two vectors. 


\section{LEAF INPUT STRUCTURE}

The input for LEAF is contained in seven cards, which are divided into three sets. The first set consists of card 0 , which establishes the print options. The second set consists of cards 1 and 2 and is used to define the decay chains. The third set is composed of four cards, which define the time-dependent case data.

The specific data for each of the three sets is detailed in Table I. Note the use of negative numbers in words 2,3 , and 4 of card 1 . If card 1 word 2 is negative, the nuclide is not retained by the filter; for example, a noble gas. If words 3 and/or 4 of card 1 are negative, then one or two branching ratio cards, card 2, must follow the card 1 on which the negative values appeared. It should also be noted that cards 5 and 6 are entered as pairs for each time interval.

Finally, we remark that the parameter IAC in word 3 card 3 controls the units used on the input of the initial concentrations and source terms.

\section{COMPARISONS}

Extensive testing of the $D(C)$ and $Z(C)$ algorithms was performed and compared with analytic solutions to validate the programming. Problems involving off-diagonal elements above and below the diagonal, as well as a constant times the identity matrix, were solved successfully.

Finally, as an independent test of the LEAF model equation solutions, several problems were solved analytically using a Laplace transform technique on MACSYMA.? We report here three such tests. These test problems are not intended to represent a real accident sequence; they were designed to test the accuracy of the LEAF solutions when compared to independently constructed analytic solutions.

The first two problems use the simple decay chain defined by ${ }^{8}$

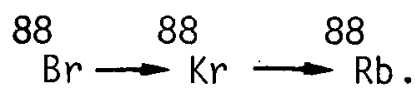

The basic data involved is given in Table II.

In these first two sample problems the source was held constant in time, either zero or a fixed value. The filtration rate and leakage rate were held constant during the course of the problem:

$$
\begin{aligned}
& V=\text { filtration rate }=2.5 \times 10^{-4} \mathrm{~s}^{-1} \\
& \bar{L}=\text { leakage rate }=1.157 \times 10^{-8} \mathrm{~s}^{-1} .
\end{aligned}
$$

The containment building inventory, filter inventory, and the integrated release were evaluated at $0,2,4,6,8$, and $24 \mathrm{~h}$. 
TABLE I

LEAF INPUT CARDS

\begin{tabular}{|c|c|c|c|c|}
\hline CARD & WORD & FORIMAT & SYMBOL & DESCRIPTION \\
\hline \multirow[t]{2}{*}{0} & 1 & 14 & NSKIP & $\begin{array}{l}\text { NSKIP }=0: \text { Unabridged output } \\
\text { NSKIP }=1: \text { Abbreviated terminal output }\end{array}$ \\
\hline & 2 & 14 & MATRIX & $\begin{array}{l}\text { MATRIX }=0: \text { Do rot print } A \text { matrix } \\
\text { MATRIX }=1: \text { Print A matrix }\end{array}$ \\
\hline \multirow{6}{*}{1} & 1. & A7 & HANMAT $(I, 1)$ & Alphanumeric nuclide name \\
\hline & 2 & $F 4$ & $\operatorname{HANMAT}(1,2)$ & $\begin{array}{l}\text { Nuclide ID No., negative if nuclide not } \\
\text { retained by filter }\end{array}$ \\
\hline & 3 & F4 & $\operatorname{HANMAT}(I, 3)$ & $\begin{array}{l}\text { Decay Parent No. 1, negative if branching } \\
\text { ratio involved }\end{array}$ \\
\hline & 4 & F4 & HANMAT $(I, 4)$ & $\begin{array}{l}\text { Decay Parent No. } 2 \text {, negative if branching } \\
\text { ratio involved }\end{array}$ \\
\hline & 5 & E 12.5 & $\operatorname{ANMAT}(1,1)$ & Nuclear decay constant $\left(\mathrm{s}^{-1}\right)$ \\
\hline & 6 & E 12.5 & $\operatorname{ANMAT}(I, 2)$ & Atomic mass in gram-atoms \\
\hline 2 & 1 & E 12.5 & $B R V(M)$ & $\begin{array}{l}\text { Branching ratio assoicated with first } \\
\text { negative decay parent, if applicable }\end{array}$ \\
\hline $2^{\prime}$ & 1 & E 12.5 & $3 R V(M+1)$ & $\begin{array}{l}\text { Branching ratio associated with second } \\
\text { negative decay parent, if applicable }\end{array}$ \\
\hline & & A BLANK & ARD MUST FOLLOW & E LAST PAIR OF CARDS 1 AND ? \\
\hline \multirow{4}{*}{3} & 1 & 14 & INT & Nuinber of time intervats \\
\hline & 2 & 14 & ITP & $\begin{array}{l}\text { PRINTING FREQUENCY } \\
\text { ITP }=1: \text { Print every interva } 1 \\
\text { ITP }=2: \text { Print every second interval } \\
\text { ITP }=\mathrm{N}: \text { Frint every Nth interval }\end{array}$ \\
\hline & 3 & 14 & IAC & $\begin{array}{l}\text { Input units of initial concentration and } \\
\text { source terms } \\
\text { IAC }=0: \text { Atoms and atoms } / s \\
\text { IAC }=1: \text { Curies and curies } / \mathrm{s} \text { if radio- } \\
\text { active, grams if stable }\end{array}$ \\
\hline & 4 & E 12.5 & TEND(INT + 1) & Time at end of problem in hours \\
\hline \multirow{4}{*}{4} & 1 & E 12.5 & COITIIC(1) & $\begin{array}{l}\text { Initial concentration (atoms) of nuciide } 1 \\
\text { in the containment building }\end{array}$ \\
\hline & 2 & E 12.5 & CONTIC (2) & $\begin{array}{l}\text { Initial concentration (atoms) of nuclide } 2 \\
\text { in the containment building }\end{array}$ \\
\hline & : & $\dot{r}$ & $\dot{0}$ & $\dot{.}$ \\
\hline & $\mathbf{I}$ & E 12.5 & CONTIC (I) & $\begin{array}{l}\text { Initial concentration (atoms) of nuciide } \\
\text { I In the containment building }\end{array}$ \\
\hline \multirow{3}{*}{5} & 1 & E 12.5 & TEND(N) & $\begin{array}{l}\text { Beginning time in hours of the Nth time } \\
\text { step. }\end{array}$ \\
\hline & 2 & E 12.5 & LAMDAV $(N)$ & Clean-up rate $\left(s^{-1}\right)$ for Nth time step \\
\hline & 3 & E 12.5 & LAMDAL $(N)$ & Leakage rate $\left(s^{-1}\right)$ for Nth tinie siep \\
\hline \multirow{4}{*}{6} & 1 & E 12.5 & $\operatorname{SOURCE}(1, N)$ & $\begin{array}{l}\text { Source term (atoms/s) for nuclide } 1 \\
\text { in Nth time step. }\end{array}$ \\
\hline & 2 & E 12.5 & $\operatorname{SOURCE}(2, N)$ & $\begin{array}{l}\text { Source term (atoms/s) for nuc?ice } 2 \text { in } \\
\text { Nth time step }\end{array}$ \\
\hline & $\dot{\bullet}$ & $\dot{\bullet} \cdot$ & $\dot{.}$ & $\dot{.}$ \\
\hline & I & E 12.5 & $\operatorname{SOURCE}(I, N)$ & $\begin{array}{l}\text { Source term (atoms/s) for nuclide I in } \\
\text { Nth time step }\end{array}$ \\
\hline
\end{tabular}


TABLE I I

BASIC DATA FOR LEAF

Tests 1 and 2

\begin{tabular}{|l|c|c|c|c|}
\hline NUCLIDE & $\begin{array}{c}\text { DECAY CONSTANT } \\
\left(\mathrm{s}^{-1}\right)\end{array}$ & $\begin{array}{c}\text { CONTAINMENT BUILDING } \\
\text { CONCENTRATION } \\
\text { At T }=0 \text { (atoms) }\end{array}$ & \multicolumn{2}{|c|}{ SOURCE (atoms $/ \mathrm{s})$} \\
\cline { 4 - 5 } & $8.359 \times 10^{-2}$ & $1.912 \times 10^{13}$ & 0 & $1 \times 10^{18}$ \\
\hline $88_{\mathrm{Br}}$ & $6.876 \times 10^{-5}$ & $1.090 \times 10^{18}$ & 0 & $2 \times 10^{18}$ \\
$88_{\mathrm{Rb}}$ & $6.527 \times 10^{-4}$ & $1.213 \times 10^{14}$ & 0 & $3 \times 10^{18}$ \\
\hline
\end{tabular}

In order to verify the LEAF results, the same two test problems were solved analytically using MACSYMA. ${ }^{7}$ The problem solved by LEAF and on MACSYMA is defined as follows.

$$
\frac{d N}{d \bar{t}}=A N+\underline{S}
$$

where

$$
\underline{N}=\left[\begin{array}{l}
N_{1} \\
N_{2} \\
N_{3} \\
F_{1} \\
F_{2} \\
F_{3}
\end{array}\right], \quad \underline{S}=\left[\begin{array}{c}
S_{1} \\
S_{2} \\
S_{3} \\
0 \\
0 \\
0
\end{array}\right] \quad, \quad \underline{N}_{0}=\left[\begin{array}{c}
1.912 \times 10^{13} \\
1.090 \times 10^{18} \\
1.213 \times 10^{14} \\
0 \\
0 \\
0
\end{array}\right]
$$

and

$$
\underline{R}(\tau)=\bar{L} \int_{0}^{\tau} d t^{\prime} \underline{N}\left(t^{\prime}\right)
$$


with

$$
R=\left[\begin{array}{l}
R_{1} \\
R_{2} \\
R_{3} \\
0 \\
0 \\
0
\end{array}\right], \quad L=\left[\begin{array}{cccccc}
1.157 \times 10^{-8} & 0 & 0 & 0 & 0 & 0 \\
0 & 1.157 \times 10-8 & 0 & 0 & 0 & 0 \\
0 & 0 & 1.157 \times 10^{-8} & 0 & 0 & 0 \\
0 & 0 & 0 & 0 & 0 & 0 \\
0 & 0 & 0 & 0 & 0 & 0 \\
0 & 0 & 0 & 0 & 0 & 0
\end{array}\right] \text {, }
$$

and the $A$ matrix given by

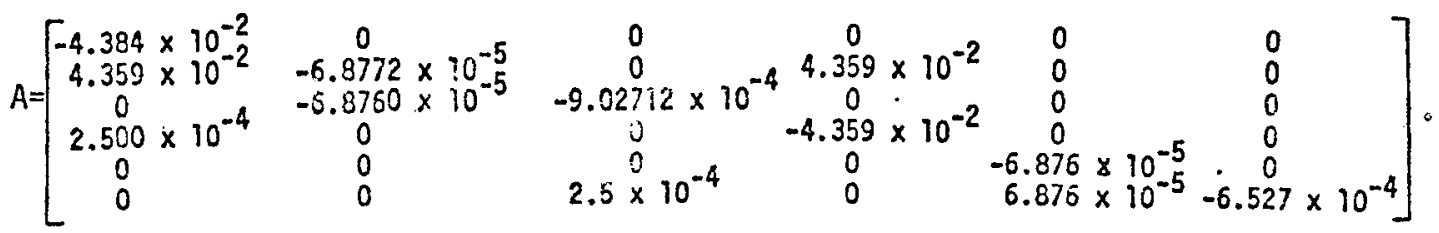

Comparisons of the LEAF and MACSYMA solutions are given in Tables III and IV. We note that in most instances six-digit agreement occurs, with the maximum discrepancy in the fifth digit.

The third test problem was the mass -85 chain defined by ${ }^{8}$

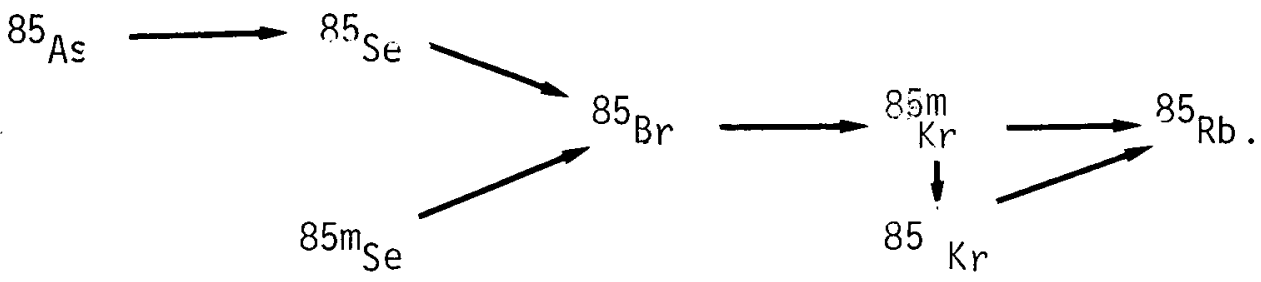

As an illustration of the LEAF program, the problem cards for Eq. (42) are listed in Table $V$ for a BATCH CROS-CDC-7600 run. In Table VI the decay cha in and nuclide data LEAF output are displayed.

Table VII lists the filtration removal rate, leakage removal rate and source terms for the various time intervals. Note in Table VII that a source to the containment building was non zero for all times. At 42 hours it was changed and again held constant.

Table VIII displays the A matrix constructed from the input (see Eq. 35) in quadrant form. Finally in Table IX the fission product inventories are given at $t=0,2,4,6,8,24,30,36,42,48,54$, and 60 hours. Note in Table IX that ${ }^{85} \mathrm{Rb}$ is stable and the listing is marked with an * indicating that the inventory is given in atoms and grams, not atoms and curies. 
TABLE III

Test Problem 1

88 CHAIN - ZERO SOURCE

(AII results in atoms)

\begin{tabular}{|c|c|c|c|c|c|c|c|}
\hline \multirow[b]{2}{*}{$\begin{array}{l}\text { TIME } \\
\text { (hours) }\end{array}$} & \multirow[b]{2}{*}{ NUCLIDE } & \multirow{2}{*}{\multicolumn{2}{|c|}{$\begin{array}{l}\text { CONTAINMENT INVENTORY } \\
\text { LEAF }\end{array}$}} & \multicolumn{2}{|c|}{ FILTER INVENTORY } & \multicolumn{2}{|c|}{ INTEGRATED RELEASE } \\
\hline & & & & LEAF & MACSYMA & LEAF & MACSYMA \\
\hline \multirow[t]{3}{*}{$\mathbf{0}$} & ${ }^{88} \mathrm{Br}$ & $1.9120 \times 10^{13}$ & $1.9120 \times 10^{13}$ & 0 & 0 & 0 & 0 \\
\hline & ${ }^{88} \mathrm{Kr}$ & $1.0900 \times 10^{18}$ & $1.0900 \times 10^{18}$ & 0 & 0 & 0 & 0 \\
\hline & ${ }^{88} \mathrm{Rb}$ & $1.2130 \times 10^{14}$ & $1.2130 \times 10^{14}$ & 0 & 0 & 0 & 0 \\
\hline \multirow[t]{3}{*}{2} & ${ }^{88} \mathrm{Br}$ & 0 & 0 & $3.77076 \times 10^{-18}$ & 0 & $5.04604 \times 10^{6}$ & $5.04604 \times 10^{6}$ \\
\hline & ${ }^{88} \mathrm{Kr}$ & $6.64341 \times 10^{17}$ & $6.64339 \times 10^{17}$ & 0 & 0 & $7.16153 \times 10^{13}$ & $7.16152 \times 10^{13}$ \\
\hline & ${ }^{88} \mathrm{Rb}$ & $5.46412 \times 10^{16}$ & $5.46410 \times 10^{16}$ & $2.24197 \times 10^{16}$ & $2.24196 \times 10^{16}$ & $4.75620 \times 10^{12}$ & $4.75619 \times 10^{12}$ \\
\hline \multirow[t]{3}{*}{4} & ${ }^{88} \mathrm{Br}$ & 0 & 0 & 0 & 0 & $5.04604 \times 10^{6}$ & $5.04604 \times 10^{6}$ \\
\hline & ${ }^{88} \mathrm{Kr}$ & $4.04900 \times 10^{17}$ & $4.04897 \times 10^{17}$ & 0 & 0 & $1.15263 \times 10^{14}$ & $1.15263 \times 10^{14}$ \\
\hline & ${ }^{88} \mathrm{Rb}$ & $3.33846 \times 10^{16}$ & $3.33844 \times 10^{16}$ & $1.42828 \times 10^{16}$ & $1.42827 \times 10^{16}$ & $8.35332 \times 10^{12}$ & $8.35329 \times 10^{12}$ \\
\hline \multirow[t]{3}{*}{6} & ${ }^{88_{\mathrm{Br}}}$ & 0 & 0 & 0 & 0 & $5.04604 \times 10^{6}$ & $5.04604 \times 10^{6}$ \\
\hline & ${ }^{88} \mathrm{Kr}$ & $2.46777 \times 10^{17}$ & $2.46774 \times 10^{17}$ & 0 & 0 & $1.41865 \times 10^{14}$ & $1.41865 \times 10^{14}$ \\
\hline & ${ }^{88} \mathrm{Rb}$ & $2.03472 \times 10^{16}$ & $2.03470 \times 10^{16}$ & $8.71126 \times 10^{15}$ & $8.71119 \times 10^{15}$ & $1.05467 \times 10^{13}$ & $1.05467 \times 10^{13}$ \\
\hline \multirow[t]{3}{*}{8} & ${ }^{88} \mathrm{Br}$ & 0 & 0 & 0 & 0 & $5.04604 \times 10^{6}$ & $5.04604 \times 10^{6}$ \\
\hline & ${ }^{88} \mathrm{Kr}$ & $1.50405 \times 10^{17}$ & $1.50403 \times 10^{17}$ & 0 & 0 & $1.58079 \times 10^{14}$ & $1.58078 \times 10^{14}$ \\
\hline & $88_{\mathrm{Rb}}$ & $1.24012 \times 10^{16}$ & $1.24010 \times 10^{16}$ & $5.30937 \times 10^{15}$ & $5.30930 \times 10^{15}$ & $1.18836 \times 10^{13}$ & $1.18835 \times 10^{14}$ \\
\hline \multirow[t]{3}{*}{24} & ${ }^{88} \mathrm{Br}$ & 0 & 0 & 0 & 0 & $5.04604 \times 10^{6}$ & $5.04604 \times 10^{6}$ \\
\hline & $88_{k r}$ & $2.86362 \times 10^{15}$ & $2.86351 \times 10^{15}$ & 0 & 0 & $1.82901 \times 10^{14}$ & $1.82900 \times 10^{14}$ \\
\hline & 88 & $2.36111 \times 10^{14}$ & $2.36102 \times 10^{14}$ & $1.01087 \times 10^{14}$ & $1.01084 \times 10^{14}$ & $1.39302 \times 10^{13}$ & $7.39301 \times 10^{13}$ \\
\hline
\end{tabular}


Test Problem 2 88 CHAIN - NON ZERO

SOURCES

(All resuits in atoms)

\begin{tabular}{|c|c|c|c|c|c|c|c|}
\hline \multirow[b]{2}{*}{$\begin{array}{l}\text { TIME } \\
\text { (hours) }\end{array}$} & \multirow[b]{2}{*}{ NUCLIDE } & \multicolumn{2}{|c|}{ CONTAINMENT INVENTORY } & \multicolumn{2}{|c|}{ FILTER INVENTORY } & \multicolumn{2}{|c|}{ INTEGRATED RELEASE } \\
\hline & & LEAF & MACSYMA & LEAF & MACSYMA & LEAF & MACSYMA \\
\hline \multirow[t]{3}{*}{0} & $88_{B R}$ & $1.91200 \times 10^{13}$ & $1.91200 \times 10^{13}$ & 0 & 0 & 0 & 0 \\
\hline & ${ }^{88} \mathrm{Kr}$ & $1.09000 \times 10^{18}$ & $1.09000 \times 10^{18}$ & 0 & 0 & 0 & 0 \\
\hline & ${ }^{88} \mathrm{Rb}$ & $1.21300 \times 10^{14}$ & $1.21300 \times 10^{14}$ & 0 & 0 & 0 & 0 \\
\hline \multirow[t]{3}{*}{2} & ${ }^{88_{B r}}$ & $2.28102 \times 10^{19}$ & $2.28102 \times 10^{19}$ & $1.30823 \times 10^{17}$ & $1.30822 \times 10^{17}$ & $1.89416 \times 10^{15}$ & $1.89416 \times 10^{15}$ \\
\hline & ${ }^{88} \mathrm{Kr}$ & $1.70224 \times 10^{22}$ & $1.70223 \times 10^{22}$ & 0 & 0 & $7.66459 \times 10^{17}$ & $7.66458 \times 10^{17}$ \\
\hline & ${ }^{88} \mathrm{Rb}$ & $4.44825 \times 10^{21}$ & $4.44824 \times 10^{21}$ & $1.57431 \times 10^{21}$ & $1.57431 \times 10^{21}$ & $2.78215 \times 10^{17}$ & $2.78214 \times 10^{17}$ \\
\hline \multirow[t]{3}{*}{4} & ${ }^{88} \mathrm{Br}$ & $2.28102 \times 10^{19}$ & $2.28102 \times 10^{19}$ & $1.30823 \times 10^{17}$ & $1.30822 \times 10^{17}$ & $3.79434 \times 10^{15}$ & $3.79434 \times 10^{15}$ \\
\hline & $88_{\mathrm{Kr}}$ & $2.74104 \times 10^{22}$ & $2.74103 \times 10^{22}$ & 0 & 0 & $2.65273 \times 10^{18}$ & $2.65273 \times 10^{18}$ \\
\hline & ${ }^{88_{R b}}$ & $5.30934 \times 10^{21}$ & $5.30933 \times 10^{21}$ & $1.97298 \times 10^{21}$ & $1.97298 \times 10^{21}$ & $6.87702 \times 10^{17}$ & $6.87702 \times 10^{17}$ \\
\hline \multirow[t]{3}{*}{6} & ${ }^{88} \mathrm{Br}$ & $2.28102 \times 10^{19}$ & $2.28102 \times 10^{19}$ & $1.30823 \times 10^{17}$ & $1.30822 \times 10^{17}$ & $5.69453 \times 10^{15}$ & $5.69453 \times 10^{15}$ \\
\hline & $88_{\mathrm{Kr}}$ & $3.37417 \times 10^{22}$ & $3.37416 \times 10^{22}$ & 0 & 0 & $5.22152 \times 10^{18}$ & $5.22150 \times 10^{18}$ \\
\hline & ${ }^{88} \mathrm{Rb}$ & $5.83137 \times 10^{21}$ & $5.83136 \times 10^{21}$ & $2.19680 \times 10^{21}$ & $2.19680 \times 10^{21}$ & $1.15352 \times 10^{18}$ & $1.15352 \times 10^{18}$ \\
\hline \multirow[t]{3}{*}{8} & $88_{\mathrm{Br}}$ & $2.28102 \times 10^{19}$ & $2.28102 \times 10^{19}$ & $1.30823 \times 10^{17}$ & $1.30822 \times 10^{17}$ & $7.59471 \times 10^{15}$ & $7.59471 \times 10^{15}$ \\
\hline & $88_{\mathrm{Kr}}$ & $3.76004 \times 10^{22}$ & $3.76003 \times 10^{22}$ & 0 & 0 & $8.20627 \times 10^{18}$ & $8.20624 \times 10^{18}$ \\
\hline & $88_{\mathrm{Rb}}$ & $6.14954 \times 10^{21}$ & $6.14952 \times 10^{21}$ & $2.33302 \times 10^{21}$ & $2.33302 \times 10^{21}$ & $1.65364 \times 10^{18}$ & $1.65364 \times 10^{18}$ \\
\hline \multirow[t]{3}{*}{24} & ${ }^{88} \mathrm{Br}$ & $2.28102 \times 10^{19}$ & $2.28102 \times 10^{19}$ & $1.30823 \times 10^{17}$ & $1.30822 \times 10^{17}$ & $2.27962 \times 10^{16}$ & $2.27962 \times 10^{16}$ \\
\hline & ${ }^{88} \mathrm{Kr}$ & $4.35080 \times 10^{22}$ & $4.35077 \times 10^{22}$ & 0 & 0 & $3.62839 \times 10^{19}$ & $3.62838 \times 10^{19}$ \\
\hline & ${ }^{88_{\mathrm{Rb}}}$ & $6.63663 \times 10^{21}$ & $6.63660 \times 10^{21}$ & $2.54156 \times 10^{21}$ & $2.54155 \times 10^{21}$ & $6.00086 \times 10^{18}$ & $6.00084 \times 10^{18}$ \\
\hline
\end{tabular}




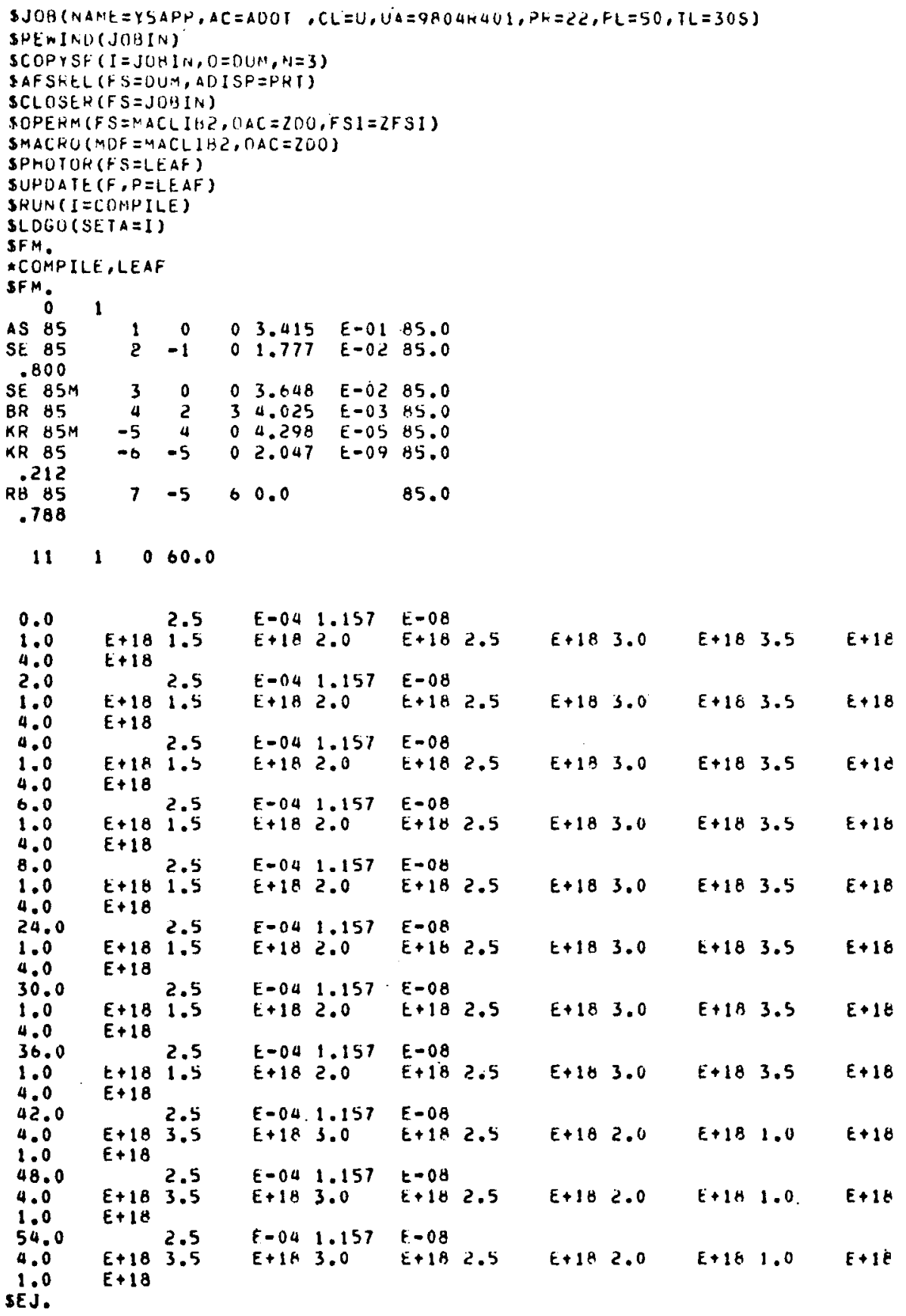


TABLE VI

DECAY CHAINS AND NUCLIDE RELATED DATA

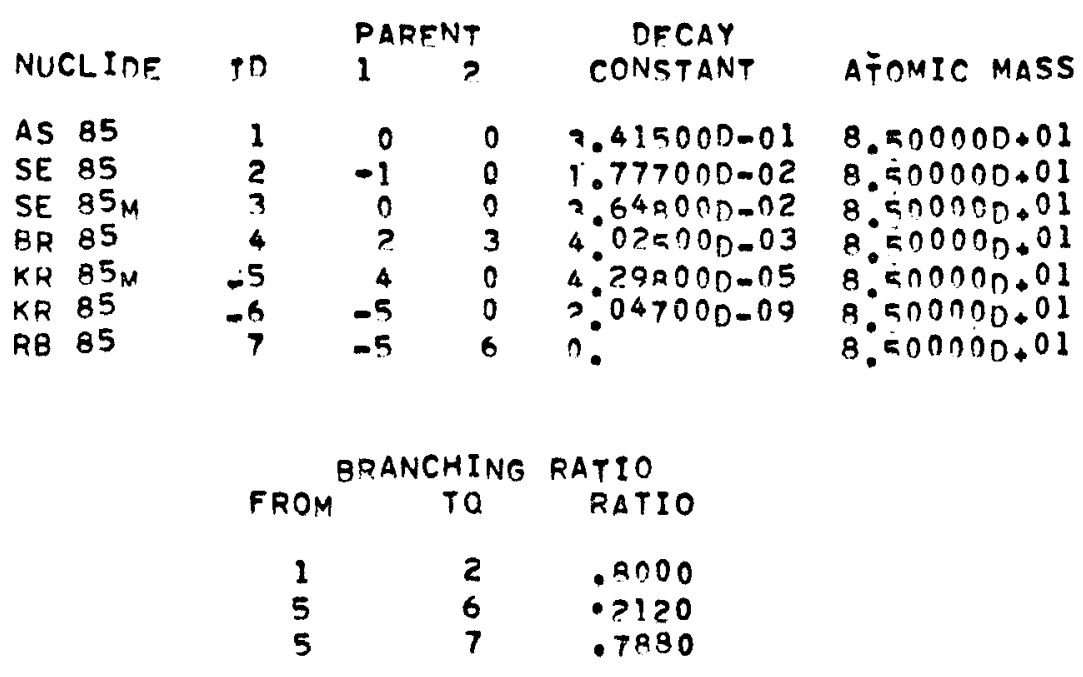


TABLE VII

FILTRATION REMOVAL RATE, LEAKAGE REMOVAL RATE, AND

SOURCE TERMS FOR TIME INTERVALS

TIME STEP DATA

TIME DERITD IN HOURS

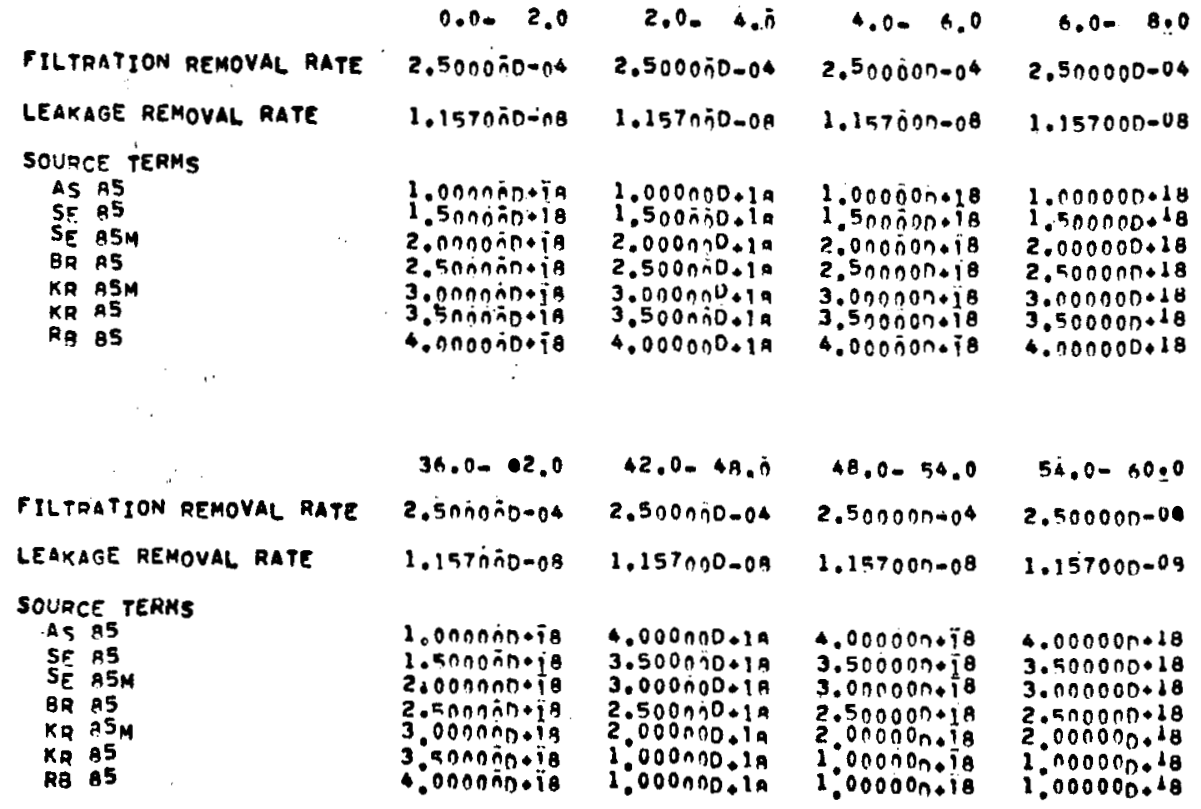


THE A MATRIX PRINTED BY QUADRANTS

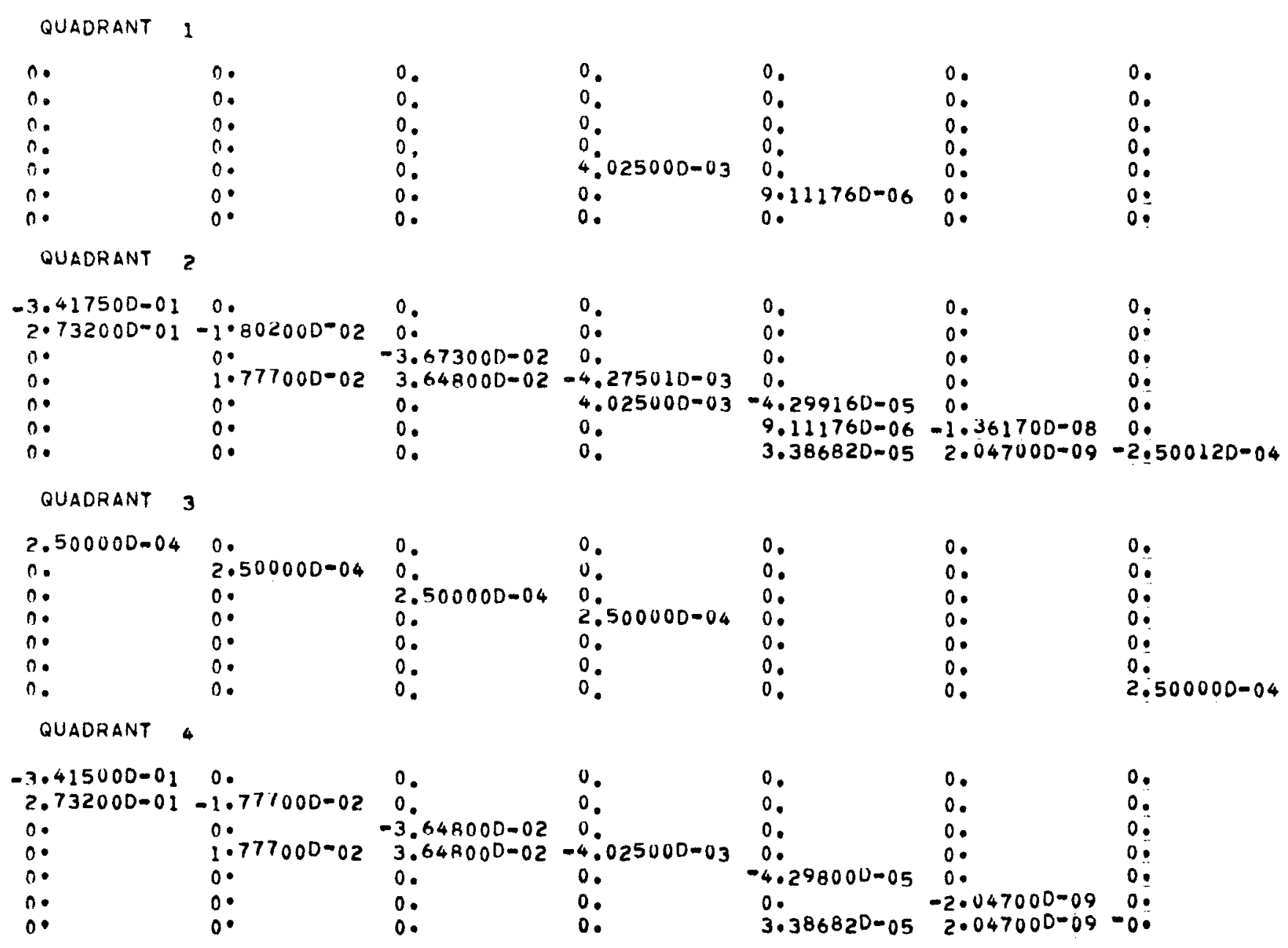


TABLE IX

FISSION PRODUCT INVENTORIES

FISSION PRODUCT INVENTORY AT 0.0 HOURS

STABLE NIJCLIDE INVENTURIES ARE GIVEN IN GRAMS AND ARE NOTED BY A H IN THE MARGIN

\section{NUCLIDE}

$\begin{array}{ll} & \\ A S & 85 \\ S E & 85 \\ S E & 85 M \\ B K & 85 \\ K K & 85 M \\ K K & 85 \\ R B & 85\end{array}$

\section{CONTAINMENT INVENTORY}

ATOMS

$$
\begin{aligned}
& -0 \\
& -0 \\
& -0 \\
& -0 \\
& -0 \\
& =0 \\
& =0
\end{aligned}
$$

CURTES,GM

0.
0
0
0
0
0

\section{FILTER INVENTORY}

ATOMS

0.
$0:$
$0:$
$0:$
0.
CURTES, GM

0.
0.
$0:$
0.

\section{INTEGRATEN RELEASE}

\section{ATOMS CUHIES,GM}

$\begin{array}{ll}0 . & 0 . \\ 0 & 0 . \\ 0 & 0 . \\ 0 & 0 . \\ 0 & 0 .\end{array}$

\section{FISSION PRODUCT INVENTORY AT ?.ON HOURS}

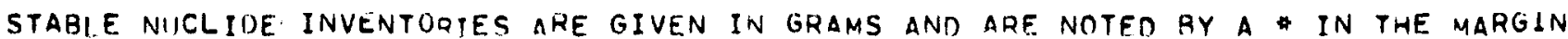

\section{NUCLIDE}
AS 85
SE $B 5$
SE $85 \mathrm{M}$
tWR 85
$K R$. $85 \mathrm{M}$
$K R \quad 85$
- Rid 85

CONTAINMENT INVENTORY

$$
\text { ATOMS }
$$

CURTES.RM

$$
\begin{aligned}
& 2.926120+18 \\
& 1.776030+20 \\
& 5.445140+19 \\
& 1.579860+21 \\
& 5.929230+22 \\
& 2.718920+22 \\
& 1.775640+22
\end{aligned}
$$

FILTER INVENTORY

$\begin{array}{cl}\text { ATOMS } & \text { CUNTES.GM } \\ 2.142110+15 & 1.977110+04 \\ 1.828140+18 & 8.780020+05 \\ 3.731590+17 & 3.679150+05 \\ 1.095810+20 & 1.19206 n+07 \\ 0 . & 0 . \\ 0 . & 0.9 \\ 1.844150+22 & 2.602780+00\end{array}$

INTEGRATEO RELEASE

ATOMS

CUKIES, GM

$2.435580+14 \quad 2.248900+03$

$1.054640+16 \quad 5.005140+03$

$\begin{array}{ll}1.054640+16 & 5.005140+03 \\ 4.518870+15 & 4.455360+03\end{array}$

$1.268390+17 \quad 1.314810+04$

$2.527470+182.935970+03$

$1.104850+18 \quad 6.112500-02$

$8.534710+17 \quad 1.204570-04$ 
FISSION PRODUCT INVENTORY AT 4.0 ON HOURS

STARLE NUCLIDE INVENTORTES ARE GIVEN IN GRAMS ANO ARE NOTED BY A * IN THE MARGIN

NUCLIDE

AS 85

SE 85

St $85 \mathrm{M}$

BK 85

$K K$ KSM

$K R \quad 85$

- RB do

\section{CONTAINMENT INVENTORY}

ATOMS

$2.926120+18$

$1.276030+20$

$5.445140+19$

$1.574860+21$

$1.041020+23$

$5.782350+22$

$2.631960+22$

\begin{abstract}
CURTES.GM
\end{abstract}
$2.70 n 73 n+07$

$6.12841 n+07$

5.358610 .07

$1.71863 n+08$

$1.2 \ln 310+08$

$3.190050+03$

$3.723140+00$
FILTER INVFNTARY

$\begin{array}{ll}\text { ATOMS } & \text { CURTES,GM } \\ 142110+15 & 1.977110+04 \\ 82.3140+18 & 8.787020+05 \\ 731590+17 & 3.679150+05 \\ 095810+20 & 1.192060+07 \\ & 0 . \\ & 0 . \\ B 8320 D+22 & 8.303390 \times 00\end{array}$

INTEGRATEN RELEASE

ATOMS CUHIES,GM

$4.874150+14 \quad 4.478710+03$

$2.117630+16 \quad 1.017040+04$

$9.054880+15 \quad 8.927630+03$

$2.584480+17 \quad 2.811490+04$

$9.433240+18 \quad 1.045790+04$

$4.625400+18 \quad 2.558970-01$

$2: 722740+18 \quad 3.842810=04$

\section{FISSTON PRODUCT TAVENTORY AT 6.00 MOUIRS}

STARLE NICLIDE INVENTORIES ARE GIVEN IN GRAMS AND ARE NOTED BY A IN THE MARGIN

NUCLIDE

AS 85

$S E 85$

SE B5M

38 .5

$K R \quad 85 M$

$K R \quad 85$

- R8 85

\section{CANTAINMENT INVENTORY}

$$
\text { ATOMS CIIZIES.QM }
$$

$>.226 i 20+18$ 1. $\operatorname{3} \sin 30+20$

$1 .=79960+? 1$

$1.2713 R 0+23$

0. กิ98र10+22

$3.798,00+22$
$2.7 n 0730+07$

$5.12841 n+07$

5. $348610+07$

$\therefore .>18+3 n+08$

$1.50303 n+08$

5.033 a 5,03

$4.513717+00$

\section{FILTER INVENTORY}

ATOMS CURIESTGM

$2.14211 n+15 \quad 1.997110+54$

$1.92814 n+18 \quad 3.780020+05$

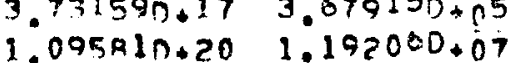

0 .

$1.11640 n+23 \quad 1.575900+n i$

\section{INTEGRATEN RELEASE}

ATOMS CURIES.GM

$7.317720 .14 \quad 6.748520 .03$

$3.18 n 520 \cdot 16 \quad 1.527560 .04$

$3.900560+17 \quad 4.243170+04$

$1.955580+19 \quad 2.271650+04$

$1.08 \pi 870+19 \quad 5.979370-01$

$5.167650+18 \quad 7.293480-04$ 
FISSTON PRODUCT INVENTORY AT 8.00 HOIIRS

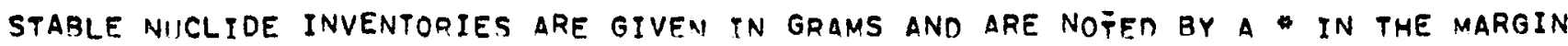

NUCLIDE

AS 85

SE 85

SE $85 \mathrm{M}$

$8 H 95$

KR $\$ 5 \mathrm{M}$

$K R \quad 85$

- RB 85

\section{CONTAINMENT INVENTORY}

ATOMS

$$
\text { CIRIES.OM }
$$

$2.026120+18$

$1 .+376 n 30+20$
$1.05140+19$

$5.145140+19$
$1.079850+21$

$1.413 ; 40+23$

1. $350,380+23$

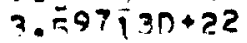

$2.70073 n+07$

$6.12841 n+07$

$5.368610+07$

$1.71863 n+08$

$1 . R+3 B A n+0 B$

$6.0713 ? n+03$

5. $176 \sin +00$
FILTFR INVENTORY

ATAMS

\section{$2.142 i 1 n+15$}

$1.8>814 n+18$

$3.731590+17$

0 .

0.

$0.73010 n+23$
CURIES,OM

$1.977110+04$

$8.780020+05$

3.679150 .15

$1: 192000+07$

0 .

$2.441810+0 i$

\section{INTEGRATEN RELEASE}

ATOMS CURIES,OM

$9.740290+14 \quad 8.998340+03$

$4.243610+16 \quad 2.038080+04$

$1.817590+16 \quad 1.787220+04$

$5.21 \times 640+17 \quad 5.674860+04$

$3.2038 B D+19 \quad 3.721700+04$

$1.982610+19 \quad 1.097420+00$

$8.00 R 890+18 \quad 1.130070-03$

FISSTON PRODUCT INVENTORY AT 24.00 HOUPS

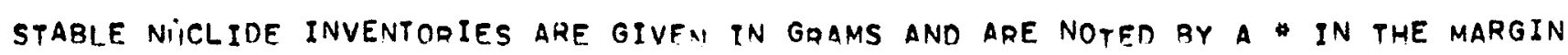

NUCLIOE

SE 85

SE $85 M$

$8 R$ 95

KR $85 M$

KR 85

- RB 85

\section{CONTAINMENT INVENTORY}

$$
\text { ATOMS }
$$

2. $026,20+18$

$1 . ? 76 n 30+20$

$5.445140+19$

$1.979 Q 4 D+21$

2. $323500+23$

$4.340920+23$

$4 . \div 96 \div 10+22$
CIRIES.OM

$2.70073 n+07$ $6.12 R 41 n+07$ 5.388410 .07

$1.71863 n+08$ $2.52287 n+08$ $2 \cdot 4 \cap 15 A \cap+04$ $6.49794 n+00$

\section{FiLTER. INVENTORY}

$$
\text { ATOMS }
$$

2. $14231 n+15$ $1.82314 n+18$

$10058 \ln +20$

$c$.

0 .

$7.90025 n+23 \quad 1.1150<0+0 ?$

\section{INTEGRATEN RELEASE}

ATOMS

CURIES:GM

$2.924990+15 \quad 2.699680+04$ $\begin{array}{ll}1.274750 .17 & 6.122250+04 \\ 5.441510+16 & 5.365030+04\end{array}$ $5.441510+16 \quad 5.365030+04$ $1.67=270+20 \quad 1.946030+05$ $2.042640+20 \quad 1.133390+01$ $3.65 \times 240+19 \quad 5.160320-03$ 
FISSTON DRODUCT INVFNTORY AT 30.00 HOIIRS

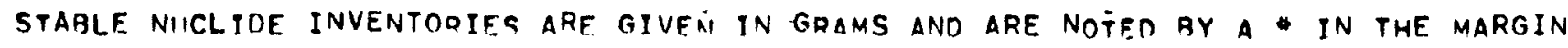

NUCLIDE

AS 85

SE 85

SE $85 \mathrm{M}$

BH 95

KR B5M

$K R \quad 85$

- RB 85

\section{CONTAINMENT INVENTORY}

ATOMS

$? .926,20+18$
$1.276 n 30+20$
$5.545,40+19$
$1.579840+21$
$2.357790+23$
$5.536920+23$
$4.952140+22$

CIJPIES.GM

$2.79073 n+07$

$6.1 \geq 841 n \cdot 07$

$5.258410+07$

$1.71863 n+08$

2. $R>?>>\cap 008$

$3.05,3>7 n+04$

$6.565010+00$
FILTER INVENTORY

ATAMS

$2.14211 n+15$

$1.8>814 n+18$

$3.73159 n+17$

0 .

$\because: 03097 n+24 \quad \dot{0} .467790+n ?$

\section{INTEGRATEN RELEASE}

AT̃OMS

CURIESIGM

$3.656 ? 60+15 \quad 3.374630+04$

$1.592650+17 \quad 7.6538 .10 .04$

$6.80>310+16 \quad 6.706710 .04$

$1.960350+18 \quad 2.142340 .05$

$2.236830+20 \quad 2.597180+15$

$3.28>800+? 0 \quad 1.816170+n$

$4.813980+19 \quad 6.792920=03$

FISSTON DRODUCT INVENTORY AT 36.00 HOURS

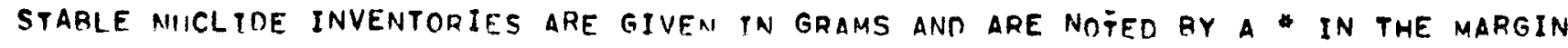

NUCLIDE

AS 85

SE 95

SE $95 \mathrm{M}$

BR 85

KR $85 M$

KR 85

- RB 85

\section{CONTA INMENT INVENTORY}

$$
\text { ATOMS }
$$

CHRIES.SM

$2.026120+18$
$1.376 n 30+20$
$5.445,40+19$
$1.579960+21$
$2.37077 n+23$
$6.736920+23$
$4.474,30+22$

2.700730 .07

6.138410 .07

$6.1 \geq 84 \ln +07$
$5.76861 n+07$

$1.71863 n+08$

$2.47777 n+08$

$3.72716 n+04$

$6.50695 n+00$
FILTER INIVENTORY

ATOMS

CURIES,GM

$2.14211 n+15$

$1.82814 n+18$

3.73159n.17

$1.095 A 1 n+20$

0 .

0.0

$1.29187 n+24 \quad 1.823310+02$

\section{INTEGRATEN RELEASE}

AT̃OMS

CURIES,GM

$4.387530+15$

$1.917540+17 \quad 9.185380 \div 04$

$8.16 ; 120+16 \quad 8.048390+04$

2. $364180+18 \quad 2.571840 .05$

$2.801900+20 \quad 3.254750+05$

$4.81 .450 .20 \quad 2.664670 .01$

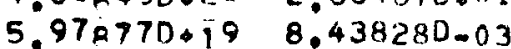


FISSTON DRODHCT INIVEJTORY AT 42.00 HOIRS

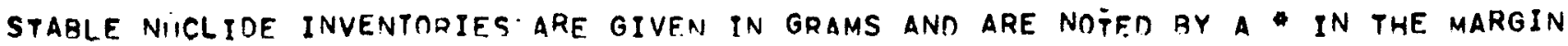

NUCLIDE

AS 85

SE 85

SE $85 M$

BR 85

KR $85 M$

$K R \quad 85$

- RB 85
CONTAINMENT INVENTORY

\section{ATOMS}

$2.02512 n+18$

1. $376 n 30+20$

$5.445140+19$

1. $579 R 60+21$

$3.376 \cap A n+23$

$7.038280+23$

4. $482 R 9 \cap \cdot 22$
CURIF.S,GM

$2.7 n n 7 ? n+07$

$6.17 .8417+07$

$5.368510+07$

$1.718 \times 3 n+08$

$2.44397 n+0 R$

$4.39180 n+04$

$6 .+0930 n+00$
FiLfFr INIVENTORY

GTOMS CURIESIGU

\section{2. $14211 n+15$}

$1.8 ? 814 n+18$

3. $73159 n+17$

$1.09591 n+20$

0 .

0.25

$1.54454 n+24$
$1.977110 . \bar{A}$

$8.78 \cap 0<0+05$

$3.679150+05$

$1.192000+0 \%$

0

$2.179930+i 2$
INTEGRATED RELEASE

ATOMS CURIESIGM

$5.118800+15 \quad 4.724510+04$

$2.23 i 440+17 \quad 1.071690 .05$

$9.523920+16 \quad 9.390070 .04$

$2.759000+18 \quad 3.001350 .05$

$3.37 n 150 \rightarrow 20 \quad 3.914840+05$

$665+190+20 \quad 3,679+70+01$

$7.149150+19 \quad 1.009870-0$ ?

\section{FISSION DRODUCT INVENTORY AT 48.00 HOIIRS}

STABLE NIICLIDE INVENTORIES ARE GIVEA IN GRAMS ANO ARE NOFED RY a IN THE MARGIN

NUCLIDE

As 85

SE 85

SE $95 M$

BR 85

KR $85 M$

KR 85

- RB 85
CANTAINMENT INVENTORY

$$
\text { ATOMS CIIRIFS.RM }
$$

$1.77045 n+19 \quad$ l.n2nว9n+08

$3.716790+20 \quad 1.7550 \mathrm{An}+08$

5. $16771 n+19 \quad 3.1529 ? n+07$

2. $26730+21 \quad 3 . n>503 n+0 R$

2. $290750+23 \quad 3.35795 n+0 R$

8. $268190+23 \quad 4.7954 ? 7+04$

$4.307560+22 \quad 5.938440+00$

\section{FILTFR INVENTORY}

ATOME CURIES,GM

A. $548420+15$

5. $36 n 76 n+18$

$5.59739 n+17$

$2.043 i 4 n+20$

0.

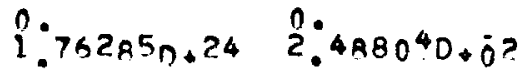

\section{INTEGRATEN RELEASE}

\section{ATOMS}

CURIES , GM

$8.043590+15 \quad 7.424020+04$ $3.15 A 700+17 \quad 1.517030+05$ $1.156430+17 \quad 1.140170 .05$ $3.461320+18 \quad 3.765360+05$ $4.025810+20 \quad 4.676470+05$ $8.72,720+20 \quad 4.1325790+0$ 8.150470+19 1.151470-0? 
FISSION PRODUCT INVFNTORY AT 54.00 HOIIRS

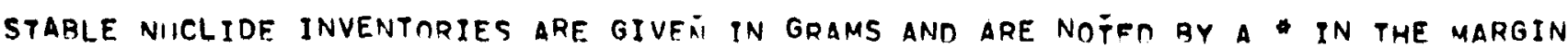

NUCLIDE

A5 85

SE 35

SE $85 M$

BR 85

KR $85 \mathrm{M}$

$K R \quad 85$

- RB 85

\section{CANTAINMENT INVENT̈IRY}

$$
\text { ATOMS CURIESIGM }
$$

\section{CURIES,GM}

$1 . \overline{1} 70450+19$

$3.716790+20$

8. $167710+19$

2. $926730+21$

3. $140100+23$

9. $478750+23$

$4.108740+22$
1. ก an? $9 n+0 B$

$1.78505 n+0 A$

$8.05202 n+07$

$3 . n 7503 n+08$

$3.6474 \ln +08$

$5.34405 n+04$

$6.5 n 466 n+00$
FiLTER INVENTORY

ATOME CUPJESIGM

$8.54842 n+i 5$

$5.36076 n+18$

$5.59729 n+17$

$2.043 i 4 n+20$

0.

$2.00245 n+24 \quad 2.826210+n \pi$

\section{INTEGRATED RELEASE}

ATOMS CURIESIGM

$1.09 \times 870+16 \quad 1.012380+05$ $4.08757 \mathrm{D}+17 \quad 1.963140+05$

$1.36 \cap 550+17 \quad 1.341430 .05$

$4.167760+18 \quad 4.533840+05$

$4.784150+20 \quad 5.557380+05$

$1.099930+21 \quad 0.079750+01$

$9.267360+191.307970-02$

\section{FISSTON DRODUCT INVFNTODY AT 60.00 HOIIRS}

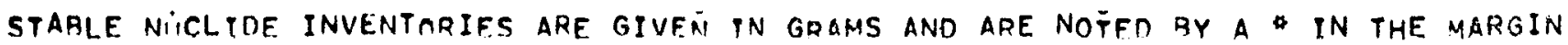

NUCLIDE

AS 85

SE 85

SE $85 \mathrm{M}$

BH 5

KR $55 \mathrm{M}$

KR 65

- RB 85

\section{CINTAINMENT INVENTODY}

ATOMS

CURIES.GM

i. $770450+19 \quad 1 . \bar{B} B 0 ? 9 n+0 B$

$3.716790+20 \quad 1.795060+08$

A. ik7710+19 $8 . n=>9 ? n+07$

?. $n 2673 n+21 \quad 3 . n>503 n+0 R$

$3.338520+23 \quad 3.74205 n+08$

$9.032100+24 \quad 5.71004 n+04$

$4.769960+22 \quad 6.73219 n+00$
FILTER INVENTORY

ATOMS CURIESTGM

8. $548 / 32 n+157.90842 D+74$

$5.36076 n+18 \quad 2.574610+n \pi$

$5.50739 n+17 \quad 5.5187<0.05$

$?: 04314 n+? 0 \quad 2.222601)+n ?$

0. 0 .

$? .226340+24 \quad 3,184540 . n>$

\section{INTEGRATEN RELEASE}

\section{ATOMS}

CURIES.GM

$1.389380+16 \quad 1.282360+05$ $5.014440+17 \quad 2.409250 .05$ $1.564670+17 \quad 1.542590+05$ $4.874190+18 \quad 5.302330 .05$ $5.587090+? 0 \quad 6.485440+05$ $1.344300 .21 \quad 7.448310 .01$ $1.044240820 \quad 1.473610-02$ 
The MACSYMA analytic solution agreed in all instances to within two digits in the sixth place. This difference is judged insignificant.

The total running time on the CDC-7600 for example 3 with LEAF was $15.3 \mathrm{~s}$; the problem solution time was $5.5 \mathrm{~s}$.

VII. CONCLUSIONS

An analytic solution has been obtained for a containment building model to calculate the leakage into the environment of each isotope of an arbitrary radioactive decay chain. The model accounts for the source, the buildup, the decay, the cleanup and the leakage of isotopes that are gas-borne inside the containment building.

Three assumptions were made in the model: (1) the gas inside the containment building is well mixed and all in one compartment; (2) that natural deposition of gas-borne isotopes internal to the containment building is ignored; and (3) that the source of an isotope inside the containment building which is a result of leakage from the reactor vessel, its removal rate by the containment cleanup system, and its leakage from the containment building are all assumed constant during short time intervals.

With these assumptions the model is representable by a system of linear differential equations. An analytic solution is obtained to these equations in terms of matrix operators using the Volterra method of the multiplicative integral. Recursion formulae are developed to accurately evaluate the matrix operators for arbitrary matrix element values.

A computer program LEAF was written, debugged, and described. Comparisons of LEAF with those achieved by Laplace transform techniques on MACSYMA ${ }^{7}$ indicate that the LEAF model is accurate. Computationally LEAF is fast. 


\section{REFERENCE}

1. "Reactor Safety Study -- Assessment of Accident Risks in Commercial Nuclear Power Plants," United States Nuclear Regulatory Commission report WASH-1400 (NUREG-75-014), Appendix VII (October 1975), p. 23.

2. John E. Foley, "131 I Release from an HTGR During the LOFC Accident," Los Alamos Scientific Laboratory report LA-5893-MS (March 1975), p. 6.

3. L. M. Carruthers and C. E. Lee, "LARC-1: A Los Alamos Release Calculation Program for Fission Product Transport in HTGRS During the LOFC Accident," Los Alamos Scientific Laboratory report LA-NUREG-6563-MS (November 1976).

4. C. E. Lee, "The Calculation of Isotopic Mass and Energy Production by a Matrix Operator Method," Los ATamos Scientific Laboratory report LA-6483-MS (September 1976).

5. F. R. Gantmacher, The Theory of Matrices, (Chelsea Publishing Company, New York, 1960), pp. 125ff, 185ff.

6. E. Bodewig, Matrix Calculus, (Interscience Publishers, New York, 1963), p. $67 f f$.

7. The MATHLAB GROUP, MASCYMA Reference Manual, Version 8, Project MAC-MIT, Cambridge, Mass. (1975) supported by the Defense Advanced Research Projects Agency work order No. 2095, under Office of Naval Research contract No. N00014-75-0661.

8. T. R. England and R. E. Schenter, "ENDF/B-IV Fission Product Files: Summary of Major Nuclide Data," Los Alamos Scientific Laboratory report LA-6116-MS (October 1975). 
APPENDIX A

$D\left(2^{p_{H}}\right)$ AND $Z\left(2^{p_{H}}\right)$ RECURSION RELATIONS

We demonstrate here an induction proof of the recursion relations for Eqs. (33) and (34) of the text.

$\underline{D\left(2^{P_{H}}\right):}$ : Define

$$
D(H)=H^{-1}\left(e^{H}-1\right)
$$

and

$$
C=2^{p_{H}} \text {. }
$$

Clearly if $p=0$

$$
D(C)=D(H) \text {. }
$$

If $p=1$

$$
\begin{aligned}
D(C) & =D(2 H)=(2 H)^{-1}\left(e^{2 H}-I\right) \\
& =H^{-1}\left(e^{H}-I\right)\left(\frac{e^{H}+I}{2}\right) \\
& =D(H)\left[I+\frac{1}{2} H D(H)\right] .
\end{aligned}
$$

By induction we may write

$$
D\left(2^{p_{H}}\right)=D\left(2^{p-1} H\right)\left[I+\frac{1}{2}\left(2^{p-1} H\right) D\left(2^{p-1} H\right)\right] \text {. }
$$

We assume Eq. $(A-5)$, which is true for $p=0$ and 1 , is true for $p=n$. Evaluate $D\left(2^{n+1} H\right)$ as

$$
\begin{aligned}
D\left(2^{n+T_{H}}\right) & =\left(2^{n+1} l_{H}\right)^{-1}\left(e^{2^{n+1} l_{H}}-I\right) \\
& =\left(2^{n_{H}}\right)^{-1}\left(e^{2^{n_{H}}}-I\right) \frac{1}{2}\left(e^{2^{n_{H}}}+I\right) \\
& =D\left(2^{n_{H}}\right)\left[I+\frac{1}{2}\left(2^{n_{H}}\right) D\left(2^{n_{H}}\right)\right] .
\end{aligned}
$$

Since Eq. $(A-5)$ is true for $p=0$ and 1 and if it is assumed true for $p=n$, it is true for $p=n+1$; then by transfinite induction it is true for all $p$.

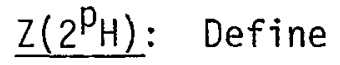

$$
H Z(H)+I=D(H) .
$$

Assume Eq. $(A-5)$ is true, as was proved. Then using Eqs. $(A-5)$ and $(A-6)$ we may write 


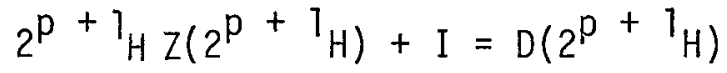

$$
\begin{aligned}
& =D\left(2^{P_{H}}\right)\left[1+\frac{1}{2}\left(2^{p_{H}}\right) D\left(2^{p_{H}}\right)\right] \text {, }
\end{aligned}
$$

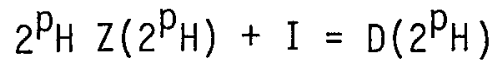

or substituting the LHS of Eq. (A-9) into the RHS of Eq. $(A-8)$ for $D\left(2^{P_{H}}\right)$ we have

or

$$
H \quad Z\left(2^{p}+1_{H}\right)=H\left\{\frac{1}{2} Z\left(2^{p_{H}}\right)+\left[\frac{1}{2}+\frac{1}{2}\left(2^{p_{H}}\right) Z\left(2^{p_{H}}\right)\right]^{2}\right\}
$$

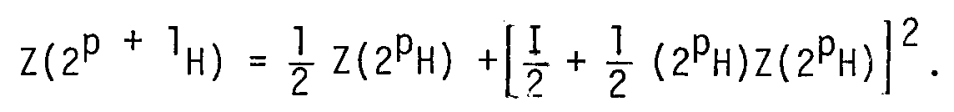

If $H$ is singular, we may define a non-singular matrix $H^{\prime}$ such that

$$
H^{\prime}=H-\varepsilon I, \quad \varepsilon \ll 1
$$

and

$$
\left|H^{\prime}\right| \neq 0
$$

which permits Eq. $(A-11)$ to be written with $H^{\prime}$, since $\left(H^{\prime}\right)^{-1}$ exists and yet is arbitrarily close to $\mathrm{H}$. The $\mathrm{H}$ matrices in LEAF will be singular if a stable isotope is in a chain.

Since $Z(H)$ exists even if $H$ is singular [see Eq. (26)], an alternate proof of the validity of Eq. (A-11) can be made by direct evaluation of the power series. That tedious process will not be repeated here; however, term by term comparison indicates that Eq. $(A-11)$ is indeed correct.

Computationaliy, Eq. (A-11) is subject to round-off errors even in double precision arithmetic. Using Eq. (A-9) in Eq. (A-11) we can eliminate that difficulty and obtain

$$
Z\left(2^{p}+7_{H}\right)=\frac{1}{2} Z\left(2^{p_{H}}\right)+\frac{1}{4}\left[D\left(2^{p_{H}}\right)\right]^{2},
$$

which involves evaluating $Z(C)$ after $D(C)$ at each point in the recursion. 


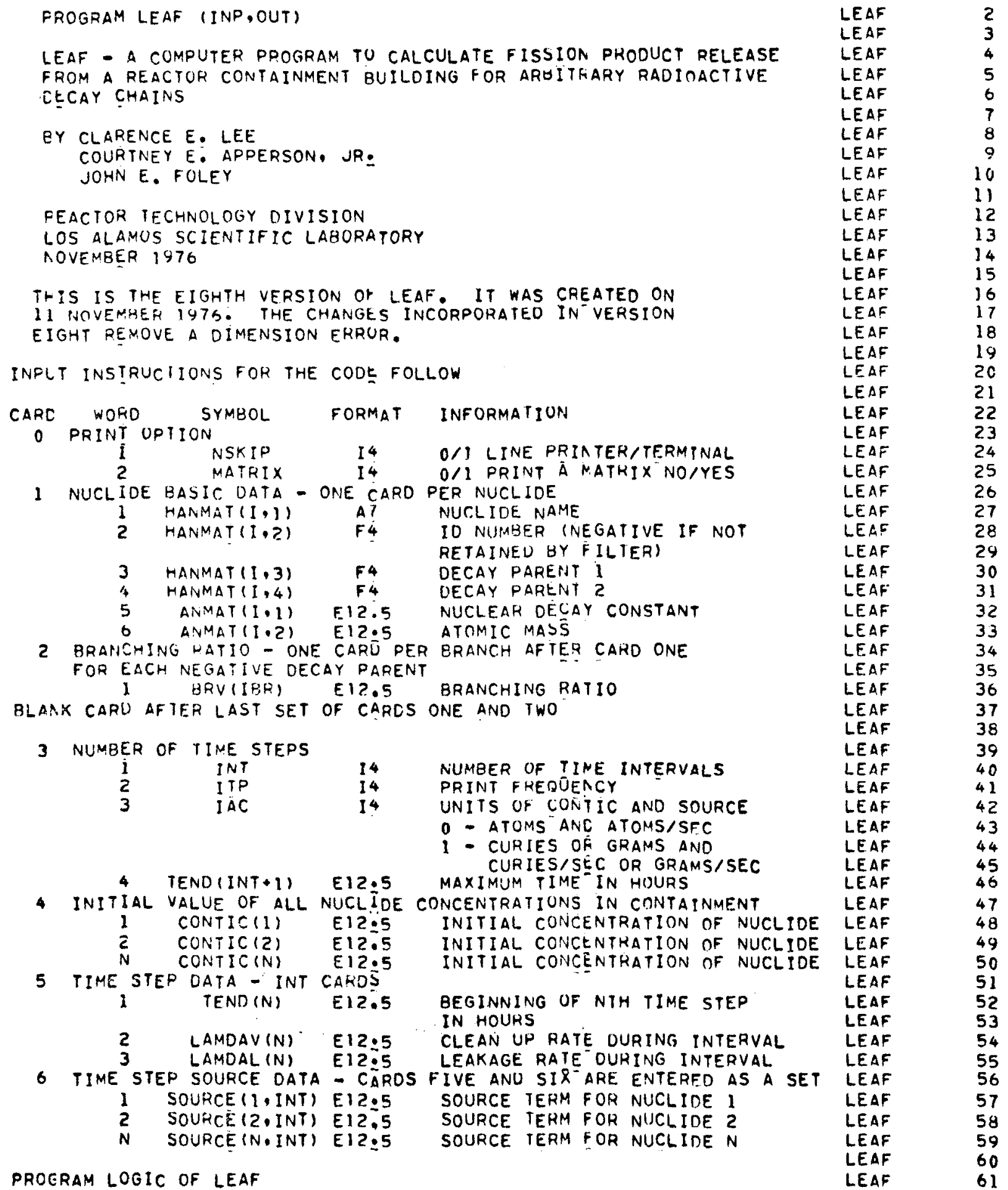




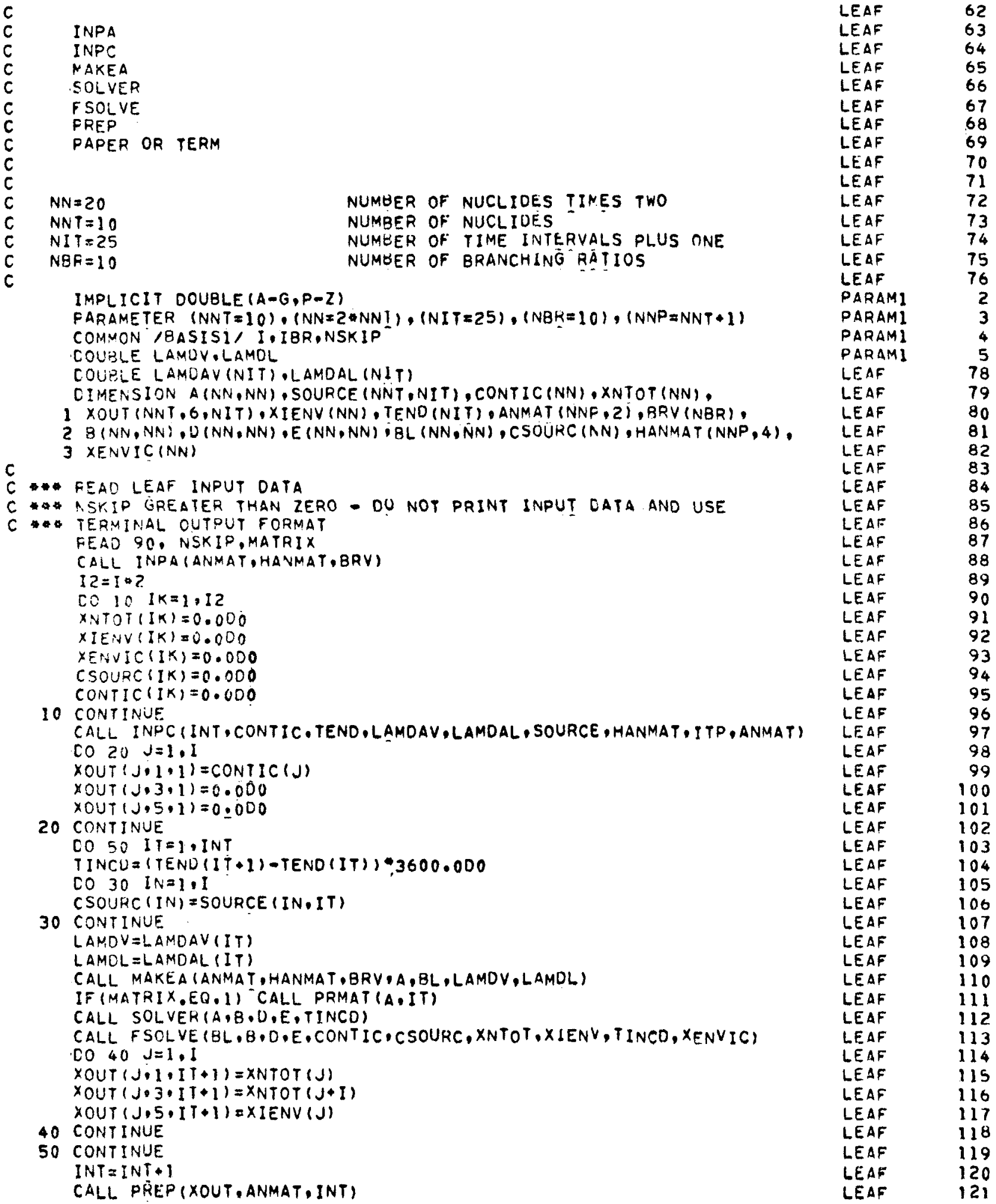


IF (NSKIP) $60,60,70$

GO CALL PAPER (XOUT, ANMAT, HANMAI, INT, ITP, TEND)

EO TO 80

70 CALL TERM (XOUT, HANMAT, INT, I!P, TEND)

c

80 CONTINÚE

SO FORMAT (2I4)

END

SUBROUTINE INPA (ANMAT, HANMAI, BRV)

C \$ INPA READS AND PRINTS THE NÜCLEAR DATA

IMPLICIT DOUELE $(A-G, P-Z)$

PARAMETER $(N N T=10),(N N=2 \$ N N I),(N I T=25),(N B R=10),(N N P=N N T+1)$

COMMON /BASISI/ I,IBR.NSKIP

CIMENSION ANMAT (NNP, 2), HANMAT (NNP, 4), BRY (NBR), HBRP $(3, N B R)$

$\vdash J=17 H \quad)$

$I=1$

$I B R=1$

C \#FEAD NUCLEAR DATA

10 FEAO 110 . (HANMAT $(I, J), J=1,4)$, (ANMAT $(I, K), K=1,2$ )

IF (HANMAT $(I, 1), E Q . H J)$ GO TO 70

CO $20 \quad J=3,4$

IF (ABS(HANMAT $(I, J))=1$ ) $20,20,30$

20 CONTINUE

CO TO 40

30 FRINT 120 . I

CALL EXIT

C * TEST FOR BHANCHING RATIOS

40 CO $60 \quad J=3.4$

IF (HANMAT(I.J) -0.0$) \quad 50.60 .00$

50 FEAD 130, BRV (IBR)

HBRP $(1, I R R)=A B S(H A N M A T(I, J))$

$+B R P(2 \cdot 1 B R)=1$

$+B R P(3, I B R)=B R V(I B R)$

$I B R=I Q R+1$

60 CONTINUE

$i=i+1$

CO TO 10

$70 \quad I=I-1$

$I B R=! B P-1$

IF (NSKIP.EQ.1) GO TO 100

C * QRINT OECAY OATA

FRINT 140

PRINT 150

FRINT $16 \mathrm{C}$

FRINT 150

FRINT I70

LCNT $=13$

CO $80 \quad \mathrm{~J}=1,1$

FRINT IBO: (HANMAT $(J, J J), J J=1,4),($ ANMAT $(J, J J), J J=1,2$ ) $L C N T=L C N T+1$ IF (LCNT.GE.60) PRINT 140

BO CONTINUE

$L C N T=L C N T+I B R+8$

IF (LCNT.GT.62) PRINT 140

C FRINT ERANCHING RATIOS

IF (IBR.EQ.0) GO TO 100

FRINT 150

FRINT 190

CO $90 \mathrm{~J}=1, \mathrm{IBR}$

FRINT $200 \cdot \operatorname{HBRP}(1, J) \cdot \operatorname{HBRP}(2 \cdot J) \cdot \operatorname{HBRP}(3 \cdot J)$

90 CONTINUE

100 CONTINLE

FETUKN

$\begin{array}{lr}\text { LEAF } & 122 \\ \text { LEAF } & 123 \\ \text { LEAF } & 124 \\ \text { LEAF } & 125 \\ \text { LEAF } & 126 \\ \text { LEAF } & 127 \\ \text { LEAF } & 128 \\ \text { LEAF } & 129 \\ \text { LEAF } & 130 \\ \text { LEAF } & 131 \\ \text { PARAM2 } & 2 \\ \text { PARAM2 } & 3 \\ \text { PARAM2 } & 13 \\ \text { LEAF } & 133 \\ \text { LEAF } & 134 \\ \text { LEAF } & 135 \\ \text { LEAF } & 136 \\ \text { LEAF } & 137 \\ \text { LEAF } & 138 \\ \text { LEAF } & 139 \\ \text { LEAF } & 140 \\ \text { LEAF } & 141 \\ \text { LEAF } & 142 \\ \text { LEAF } & 143 \\ \text { LEAF } & 144 \\ \text { LEAF } & 145 \\ \text { LEAF } & 146 \\ \text { LEAF } & 147 \\ \text { LEAF } & 148 \\ \text { LEAF } & 149 \\ \text { LEAF } & 150 \\ \text { LEAF } & 151 \\ \text { LEAF } & 152 \\ \text { LEAF } & 153 \\ \text { LEAF } & 154 \\ \text { LEAF } & 155 \\ \text { LEAF } & 156 \\ \text { LEAF } & 157 \\ \text { LEAF } & 158 \\ \text { LEAF } & 159 \\ \text { LEAF } & 160 \\ \text { LEAF } & 161 \\ \text { LEAF } & 162 \\ \text { LEAF } & 163 \\ \text { LEAF } & 164 \\ \text { LEAF } & 165 \\ \text { LEAF } & 166 \\ \text { LEAF } & 167 \\ \text { LEAF } & 168 \\ \text { LEAF } & 169 \\ \text { LEAF } & 170 \\ \text { LEAF } & 171 \\ \text { LEAF } & 172 \\ \text { LEAF } & 173 \\ \text { LEAF } & 174 \\ \text { LEAF } & 175 \\ \text { LEAF } & 176 \\ \text { LEAF } & 177 \\ \text { LEAF } & 178 \\ \text { LEAF } & 179 \\ \text { LEAF } & 180 \\ \text { LEAF } & 181 \\ \text { LEAF } & 182 \\ & \end{array}$


C

110 FORMAT $(47,3 F 4.2012 .5)$

120 FORMAT (1H0.4X. \$HERE IS AN ERROR IN NUCLIUE * I3)

130 FORMAT $(6012.5)$

140 FORMAT $(1 \mathrm{H} 1)$

150 FORMAT $(/ / 1 / 1)$

160 FORMAT $151 X$, WECAY CHAINS AND NUCLIDE RELATEO DATA\$)

170 FORMAT $161 X$, \#PAFENT*,7X, *OECAY*,1,45X,*NUCLIUE ID*,4X,*I $1 X$, CONSTANIO, $4 X$, *ATOMIC MASS*, 11

180 FORMAT $\left(45 x, A 7,1 X, F 4,2 X, F 4,1 x_{1}, F 4,2 x_{1}, 1 P D 12,5,1 x_{1}, 1 P D-12,5\right)$

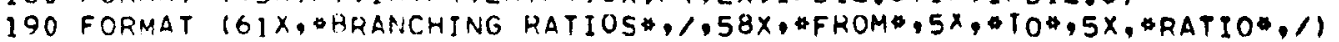

200 FOPMAT $(57 \times, F 4,4 X, F 4,3 X, F 7.4)$

ENO

SUBPOUTINE INPCIINT, CONTIC, IEND, LAMDAV, LAMDAL, SOURCE, HANMAT, ITP, AN (NAT)

C \# INPC REAUS. THE CASE DATA

IMPLICITT DOUBLE $(A-G, P-Z)$

FARAMETER $(N N T=10),(N N=2 * N N I),(N I T=25),(N B R=1 U),(N N P=N N T+1)$

COMMON /GASISI/ I, IHR,NSKIP

COUHLE PRECISION LAMDAV (NIT), LAMDAL (NIT)

CIMENSION SOURCE(NNT, NIT), HANMAT (NNP, 4), CONTIC(NN), TEND(NIT)

CIMENSION HTENI) (8), ANMAT (NNP, 2)

C * FEAI BASIC TIME DATA

FEAD IZO, INT ITP, IAC,HTIME

INT $1=I N T+1$

TEND (INTI) = HTIME

C * FEAD INITIAL CONCENTRATION UF CONTAINMENT

FEAO 130. (CONTIC (J) .J $=1$. I)

C \$ FEAO TIME STEP DATA

CO $10 \mathrm{~J}=$ ?. DNT

FEAD 130, IEND (J) , LAMUAV (J), LAMUAL (J)

FEAD 130, (SOURCE $(M, J), M=1, I)$

10 CONTINUE

C CONVEFT CONTIC ANO SOURCE TU ATOMS ANO ATOMS/SEC

IF (IAC.EO.D) GO TO GO

CO $50 \quad M=1, I$

IF (ANMAT $(M, 1)) 20.20 .30$

$20 C F=6.02250+23 /$ ANMAT $(M, 2)$ CO TO 40

$30 \mathrm{CF}=3.70+10 / \operatorname{ANMAT}(\mathrm{M}, ?)$

40 CONTINUE

CONTIC $(M)=$ CONTIC $(M) \backsim C F$

[O $50 \quad j=1$, INT

SOURCE $(M \cdot J)=$ SOURCE $(M, J) * C F$

50 CONTINUE

60 CONTINUE

C * FRINT INPUT DATA

IF (NSKIP.EQ.1) GO TO 110

FRINT 140

FRINT 160

PPINT 170

PRINT 180

LINI $=1$

$L I N Z=M I N O(7, I N T)$

LIN $2 P=L I N 2+1$

LIN $=I N T / 7.0+0.99$

LNCT $=9$

$A L N=S+1$

CO $100 \quad J K=1$, LIN

PRINT 180

$L=L I N 2 P-L I N I+1$

$L=M I N O(B \cdot L)$

CO $70 \mathrm{~J}=\mathrm{L}, \mathrm{L}$

LEAF

LEAF

LEAF

LEAF

LEAF

LEAF

LEAF

2\%.5 LEAF

LEAF

LEAF

LEAF

LEAF

LEAF

LEAF

LEAF

PARAMZ

PARAM2

PARAMZ

LEAF

LEAF

LEAF

LEAF

LEAF

LEAF

LEAF

LEAF

LEAF

LEAF

LEAF

LEAF

LEAF

LEAF

LEAF

LEAF

LEAF

LEAF

LEAF

LEAF

LEAF

LEAF

LEAF

LEAF

LEAF

LEAF

LEAF

LEAF

LEAF

LEAF

LEAF

LEAF

LEAF

LEAF

LEAF

LEAF

LEAF

LEAF

LEAF

LEAF

LEAF

LEAF

LEAF

LEAF
183

184

185

186

187

188

189

190

191

192

193

194

195

196

197

198

202

203

204

205

206

207

208

209

210

211

212

213

214

215

216

2.17

218

219

220

221

22.2

223

224

225

226

227

228

229

230

231

232

233

234

235

236

237

238

234

240

241

242

243 
$K=L I N I-1+J$

$H T E N D(J)=T E N D(K)$

70 CONTINUE

$L M=L-1$

FRINT $200,($ HTEND (J),J $=1, L M)$

FRINT 210. (HTENO (J),J J $2 \cdot L$ )

FRINT 220, (LAMDAV (J), J=LINI, LINZ)

FRINT 230 , (LAMDAL (J), J=LIN1,LIN2)

FPINT 240

CO $80 \mathrm{JL}=\mathrm{I}, \mathrm{I}$

FRINT 250 , HANMAT $(J L, 1),(S O U R C E(J L, J), J=L I N I, L I N Z)$

80 CONTINUE

$L I N I=1 . I N I+7$

LIN $=L I N 2+7$

LINZ=MIND (LIN2, INT)

$L I N>P=L I N Z+1$

FRINT 150

$L N C T=L N C T+N L N+3$

LNTST $=60-\angle N C T$

IF (LPTSTT-NLN) 90.100 .100

90 FRINT 140

LNCT $=0$

100 CONTINUE

110 CONTINUE

FETURN

120 FORMAT $(314, E) 2.5)$

130 FORMAT $(6012.5)$

140 FORMAT (IHI)

150 FORMAT $(/ / 1)$

160 FORMAT $(/ / / / /)$

170 FORMAT $(62 x$, \#TIME STEP DATA, 1$)$

180 FORIAT $159 x_{\text {. }}$ TIME PERIOD IN HOURS*, $/ 1$

190 FORMAT $\left(23 x_{0} 7\left(12 x_{0},-\#\right)\right)$

200 FORMAT $(1,+, 21 \times, 7(8 \times, F 6.1))$

210 FORMAT $(1 H+, 27 X, 7(8 X, F 6,1))$

220 FORMAT $(1,5 x$, OFILTHATION REMOVAL RATE, $7(2 \times, 1 F 012.5)$ )

230 FOFMAT $1 / .5 x$, LEAKAGE REMOVAL RATE $\$, 7(2 \times, 1 F D 12.5)$

240 FORMAT $\left(/, S x_{\text {. }}\right.$ SOURCE TERMS $\$$ )

250 FORMAT $(7 X, A 7,14 X .7(2 X, 1 P O 12.5))$

END

SUBROUTINE MAKEA (ANMAT.HANMAT, BRV, A, BL, LAMUV, LAMUL)

C * MAKEA CONSIRUCTS THE MAIN SOLUTION MATRIX

IMPLICIT DOUHLE $(A-G, P=Z)$

FARAMETER (NNT $=12),(N N=2 \$ N N 1),(N I T=25),(N B K=10),(N N P=N N T+1)$

COMMON /BASISI/ I. IBR.NSKIP

COUBLE LAMUV.LAMOL

CIMENSION ANMAT (NNP, Z), BRV (NBR), A (NN,NN), BB (NNT, NNT), BL (NN, NN)

CIMENSION HANMAT (NNP, 4)

$I 2=I * 2$

$I P=I+1$.

CO 10 IK $=1,1$ ?

$0010 \mathrm{JK}=1.12$

$A(I K \cdot J \dot{K})=0.000$

$\mathrm{EL}(I K, J K)=0.0 \mathrm{D}_{0}$

10 CONTINUE

CO 20 I $K=1$, I

CO $20 \quad J K=1$, I

$E B(I K \cdot J K)=0.000$

20 CONTINUE

$I B R=1$

CO $70 \quad I K=1$, I

CO $60 \mathrm{JK}=1,1$

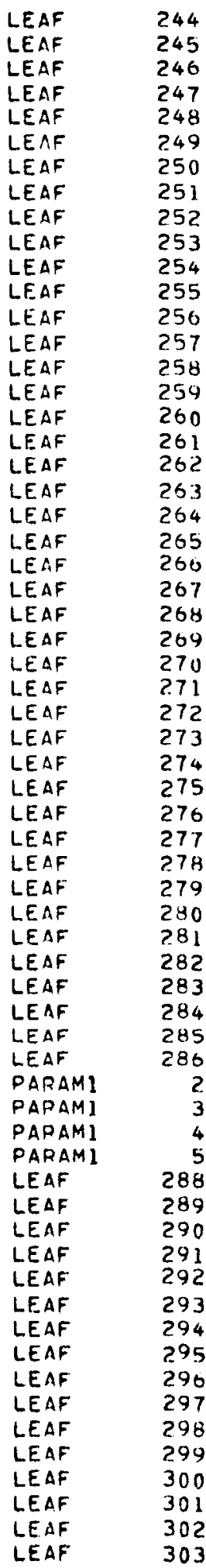


CO 50 IOX $=3,4$

C * IDENTIFY SOURCE TERMS

IF (ABS(HANMAT(IK,IDX)), NE. JK) GO TO 50

IF (HANMAT(IK,IDX)) $30,30,40$

$30 \mathrm{~EB}(I K \cdot J K)=B R V(I B R) \cdot A N M A T(J K, I)$

$I B R=I B R+1$

.60 TO 50

$40 \quad B B(I K \cdot J K)=A N M A T(J K, 1)$

50 CONTINUE

$A(I K, J K)=B B(I K, J K)$

60 CONTINUE

BH(IK,IK) =ANMAT $(I K, I)$

$A(I K \cdot I K)=-B B(I K \cdot I K)-L A M O L$

IF (HANMAT $(I K, 2) \cdot G T \cdot 0.0) \quad A(I K, I K)=A(I K, I K)-L A M D V$

70 CONTINUE

CO 80 IK $=I P, I 2$

$\checkmark K=I K-1$

IF (HANMAT $(J K, 2), G T, 0.0) \quad A(\$ K, J K)=$ LAMOV

BO CONTINUE

CO $130 \quad I K=1$, I

$J J=1 K-1$

$I L=I+I K$

If $(J J \cdot E Q .0)$ GO TO 120

$\mathrm{CO} 110^{-} \mathrm{JK}=1 . \mathrm{JJ}$

$\checkmark L=I+J K$

IF (MANMAT(IK,?)) $90,90,160$

$90 A(I K, J L)=B H(I K, J K)$

GO TO 110

$100 A(I L, J L)=B B(I K, J K)$

110 CONTINUE

120 CONTINUE

$A(I L \cdot I L)=-B B(I K, I K)$

130 CONTINUE

CO $140 \quad J=1, I$

GL $(J \cdot J)=L A M O L$

140 CONTINUE

FETURN

END

SUBROUTINE SOLVER $(A, B, D, E, T I N C D)$

$C \nleftarrow$ SOLVER EVALUATES D (A), I+A\#D(A), AND Z(A)

IMPLICIT DOUBLE $(A-G, P-Z)$

PARAMETER (NNT $=10),(N N=2 \& N N T),(N I T=25),(N B K=10),(N N P=N N T+1)$

COMMON /BASISI/ I, IEBR,NSKIP

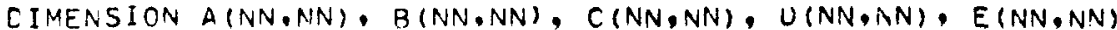
CIMENSION F(NN,NN), G(NN,NN)

$N=2$ ?

$S U M=0.000$

$I Z=1 \% ?$

CO $20 \quad J=1,12$

[O :O JJ $J=1,12$

$S U M=S U M+A(J, J J) * A(J, J J)$

10 CONTINUE

20 CONTINUE

$F=(O L O G(S U M)+2.00 \cap * D L O G(T I N C D)) /(2.000 * D L O G(2.000))$

If (P) $30,30.40$

$30 \quad A P=1$

GO TO 50

$40 \quad \wedge P=P+1 \cdot 000$

50 CONTINUE

$T=T I N C D /(2 \cdot 000 * N D)$

CALL SCALAK $(A, T, C)$

CO $70 \mathrm{~J}=1, I$ ?

CO $60 \quad J J=1,12$

\begin{tabular}{lr} 
& \\
LEAF & 304 \\
LEAF & 305 \\
LEAF & 306 \\
LEAF & 307 \\
LEAF & 308 \\
LEAF & 309 \\
LEAF & 310 \\
LEAF & 311 \\
LEAF & 312 \\
LEAF & 313 \\
LEAF & 314 \\
LEAF & 315 \\
LEAF & 316 \\
LEAF & 317 \\
LEAF & 318 \\
LEAF & 319 \\
LEAF & 320 \\
LEAF & 321 \\
LEAF & 322 \\
LEAF & 323 \\
LEAF & 324 \\
LEAF & 325 \\
LEAF & 326 \\
LEAF & 327 \\
LEAF & 328 \\
LEAF & 329 \\
LEAF & 330 \\
LEAF & 331 \\
LEAF & 332 \\
LEAF & 333 \\
LEAF & 334 \\
LEAF & 335 \\
LEAF & 336 \\
LEAF & 337 \\
LEAF & 338 \\
LEAF & 339 \\
LEAF & 340 \\
LEAF & 341 \\
LEAF & 342 \\
LEAF & 343 \\
PARAM2 & 2 \\
PARAM2 & 3 \\
PARAM2 & 3 \\
LEAF & 345 \\
LEAF & 346 \\
LEAF & 347 \\
LEAF & 348 \\
LEAF & 349 \\
LEAF & 350 \\
LEAF & 351 \\
LEAF & 352 \\
LEAF & 353 \\
LEAF & 354 \\
LEAF & 355 \\
LEAF & 356 \\
LEAF & 357 \\
LEAF & 358 \\
LEAF & 359 \\
LEAF & 360 \\
LEAF & 361 \\
LEAF & 362 \\
LEAF & 363 \\
LEAF & 364 \\
& \\
\hline
\end{tabular}


$\theta(7.7)=0.000$

60 CONTINUE.

$B(J, J)=1 \cdot n \cup 0$

70 CONTINUE

$C \nleftarrow$ CALCULATE U(H) AND $Z(H)$

CO $90 \quad J=1, M$

$F M=1.000 /(M+2.000-J)$

CALL SCALAF $(B, F M, D)$

CALL MULTI $(C, D, E)$

CO $80 \quad J J=1,15$

$E(J) \cdot J j)=E(J j, J j)+1.000$

80 CONTINUE

CALL EQUAL (E,B)

90 CONTINUE

$S=1.000$

$\begin{array}{lll}C O & 120 \quad J=1, N P\end{array}$

$G=S / 2.000$

$5=5 * 2.000$

CALL SCALAH $(C, Q, F)$

CALL MULTI $(F, H, E)$

CO $100 \quad J J=1,12$

$e(97 \cdot j u)=E(J j, 7 j)+1.000$

100 CONTINUE

CALL MULTI $(B, E, F)$

CALL EUUAL (B.B)

$C * C(A)$

CALL EQUAL $(F, B)$

CALL EQUAL (G.E)

CALL MULTI $(G, E, F)$

CALL SCALAF(F, $0.2500, G)$

CALL SCALAH $(0.0 .5000 .0)$

CO $110 \quad J I=1,12$

CO $110 \quad J j=1,12$

$110 E(J I \cdot J)=E(J I, J J)+G(J I, J J)$

$C \otimes 2(A)$

120 CONTINUE

CALL SCALAK(A.TINCO,F)

CALL MULTI $(F, B, E)$

CO $130 \mathrm{JJ}=1,12$

$130 \varepsilon(J \jmath, J)=E(J j \cdot J)+1.000$

$C * I+A \&$ D (A)

FETURN

END

LEAF $\quad 365$

LEAF $\quad 366$

LEAF 367

LEAF 368

LEAF $\quad 369$

LEAF $\quad 370$

LEAF 371

LEAF 372

LEAF $\quad 373$

LEAF $\quad 374$

LEAF 375

LEAF 376

LEAF $\quad 377$

LEAF 378

LEAF $\quad 379$

LEAF 380

LEAF 381

LEAF 382

LEAF 383

LEAF 384

LEAF $\quad 385$

LEAF 386

LEAF 387

LEAF 388

LEAF 389

LEAF 390

LEAF 391

LFAF 392

LEAF 393

LEAF $\quad 394$

LEAF 395

LEAF $\quad 396$

LEAF 397

LEAF 398

LEAF 399

LEAF $\quad 400$

LEAF 401

LEAF $\quad 402$

LEAF $\quad 403$

LEAF $\quad 404$

LEAF $\quad 405$

LEAF 406

SUGROUT INE FSOLVE TBL, O OE CONTIC CSOURC XNTOT,XIENV,TINCD XENUICI LEAF 407

C * FSOLVE CALCULATES THE FINAL CONCENTRATIONS ÁNL INTEGRALS IMPLICIT DOUHLE $(A-G, P-Z)$

FARAMETEK (NNT $=10),(N N=2 \% N N I),(N I T=25),(N 8 H=10),(N N P=N N T+1)$

COMMON /EASISI, I, IBR,NSKIP-

CIMENSION $B(N N, N N), B L(H N, N N), D(N N, N N), E(N N, N N), F(N N, N N), X(N N)$

1. $Y(N N)$, XENVIC (NN)

CIMENSION CONTIC(NN), CSOURC (NN), XNTOT (NN), XIENV (NN)

$12=I * 2$

C * CALCULATE CONTAINMENT AND FILTER INVENTURY

CALL MVMUL (E, CONTIC,X)

CALL SCALAH (B,TINCU,F)

CALL MVMUL (F, CSOURC, Y)

CALL VAUD $(X, Y, X N T D T)$

C * CALCULATE INTEGRATED RELEASE

TINCU? = TINCD TINCD

CALL SCALAH(B, TINCD,F)

CALL MVMUL (F, CONTIC, XIENV)

CALL SCALAR(U,TIVCDZ,F)

CALL MVMUL $(F, C S O U R C, Y)$

LEAF

PARAMZ

PARAM2

PARAM?

408

409

LEAF

LEAF

LEAF

LEAF

LEAF

LEAF

LEAF

LEAF

LEAF

LEAF

LEAF

LEAF

LF.AF

LEAF

LEAF

2
3
4
411
412
413
414
415
416
417
418
419
420
421
422
423
424
425 
CALL VADD $(X I E N V, Y, X)$

CALL MVMUL $(B L, X, Y)$

CALL VAOU $(Y, X E N V I C, X I E N V)$

CO $10 \mathrm{~J}=1.12$

$\operatorname{CONTIC(J)=XNTOT(J)}$

$X E N V I C(J)=X I E N V(J)$

10 CONTINUE

FETURN

END

SUHROUTINE PREP (XOUT, ANMAT, INT)

C * FREP CONVEHTS OENSITIES TO CURIES OR GRAMS

IMPLICIT DOUBLE (A-G,P-Z)

PARAMETER (NNT $=10),((N N=2 \$ N N),(N I T=25),(N B H=10),(N N P=N N T+1)$

COMMON IBASISI, I.IBR,NSKIP

CIMENSION XOUT (NNT, G,NIT), ANMAT (NNP, Z)

CO $50 \quad J=1$, INT

CO $40 \mathrm{JJ}=1$. I

IF (ANMAT $(J J \cdot 1) \cdot E Q \cdot 0.0) \quad$ GO 1020

CURIES = ANMAT $(\mathrm{J}, 1) / 3.70+10$

CO $10 \mathrm{~J} X=1.5 \cdot 2$

XOUT $(J J, J X+1, J)=X O U T(J J, J X, J) *$ CURIES

10 CONTINUE

CO $\mathrm{TO} 40$

20 CRA'AS $=A N M A T(J), 2) / 6 . n 2250+23$

CO $30 J X=1,5,2$

XOUT $(J J, J X+1, J)=X O U T(J J, J X, J)$ GRAMS

30 CONTINUE

40 CONTIINUE

50 CONTINUE

FETURT

END

SHGQOUTIUE PAPER (XOUT, ANMAT, HANMAT, INT, ITP,TENU)

C FAPE PPINIS THE RESULTS OF LEAF

IMPLICIT DUUULE $(A-G, P-Z)$

FARAMETER (NNT=12), (NN=2*NNI), (NIT $=25),(N B K=10),(N N P=N N T+1)$

COMMON /HASISI, I, IRR, NSKIP

CIMENSION XOUT (NNT, 6.NIT), HANMAT (NNP, 4), TEND (NIT), ANMAT (NNP, Z)

CO $30 \mathrm{~J}=1$, INT, ITP

PTIME $=$ TEND(J)

FRINT 40

FRINT 50 . HTIME

FRINT 60

FRINT 70

FRINT 80

CO $20 \quad J K=1 . I$

IF (ANMAT $(J K, 1), E Q .0 .0)$ GO 1010

FRINT 90, HANMAT' $(J K, 1),(X O U I(J K, J T, J), J T=1,6)$

CO TO 20

10 FRINT 100, MANMAT $(J K, 1),(X O U T(J K, J T, J), J I=1,6)$

20 CONTINUE

30 CONTINUE

C

FETURN

40 FORMAT (IHI)

50 FORMAT $(47 X, \$ F$ ISSION PRODUCI INVENTORY AT \#,F, 2 , \# HOURS, 1$)$

60 FORMAT (29X,80HSTABLE NUCLIUE INVENTORIES ARE GIVEN IN GRAMS AND IFE NOTEO GY A * IN THE MARGIN, /1)

70 FORMAT $(2 n X$, \#UCLIOE ,9X, \#UNTAINMENT INVENTOFY $10 X, \$ F I L T E R$ INVEN

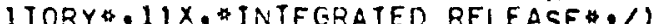

80 FORMAT $\left(3 ., x_{1}, 3(8 \times, * A T O M S\right.$ CURIES,GM\$), 1$)$

90 FORMAT (20X,A7,4X,3(3X,1PD12.5,1X,1PU12.5))

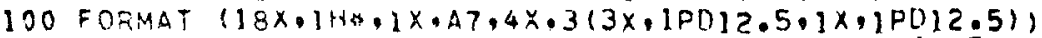
ENO

$\begin{array}{lr}\text { LEAF } & 426 \\ \text { LEAF } & 427 \\ \text { LEAF } & 428 \\ \text { LEAF } & 429 \\ \text { LEAF } & 430 \\ \text { LEAF } & 431 \\ \text { LEAF } & 432 \\ \text { LEAF } & 433 \\ \text { LEAF } & 434 \\ \text { LEAF } & 435 \\ \text { LEAF } & 436 \\ \text { PARAM2 } & 2 \\ \text { PARAM2 } & 3 \\ \text { PARAM2 } & 4 \\ \text { LEAF } & 438 \\ \text { LEAF } & 439 \\ \text { LEAF } & 440 \\ \text { LEAF } & 441 \\ \text { LEAF } & 442 \\ \text { LEAF } & 443 \\ \text { LEAF } & 444 \\ \text { LEAF } & 445 \\ \text { LEAF } & 446 \\ \text { LEAF } & 447 \\ \text { LEAF } & 448 \\ \text { LEAF } & 449 \\ \text { LEAF } & 450 \\ \text { LEAF } & 451 \\ \text { LEAF } & 452 \\ \text { LEAF } & 453 \\ \text { LEAF } & 454 \\ \text { LEAF } & 455 \\ \text { LEAF } & 456 \\ \text { PARAM2 } & 2 \\ \text { PARAM? } & 3 \\ \text { PARAM2 } & 4 \\ \text { LEAF } & 458 \\ \text { LEAF } & 459 \\ \text { LEAF } & 460 \\ \text { LEAF } & 461 \\ \text { LEAF } & 462 \\ \text { LEAF } & 463 \\ \text { LEAF } & 464 \\ \text { LEAF } & 465 \\ \text { LEAF } & 466 \\ \text { LEAF } & 467 \\ \text { LEAF } & 468 \\ \text { LEAF } & 469 \\ \text { LEAF } & 470 \\ \text { LEAF } & 471 \\ \text { LEAF } & 472 \\ \text { LEAF } & 473 \\ \text { LEAF } & 474 \\ \text { LEAF } & 475 \\ \text { LEAF } & 476 \\ \text { LEAF } & 477 \\ \text { LEAF } & 478 \\ \text { LEAF } & 479 \\ \text { LEAF } & 480 \\ \text { LEAF } & 481 \\ \text { LEAF } & 482 \\ \text { LEAF } & 483 \\ \text { LEAF } & 484 \\ \text { LEA } & \\ \text { LEA } & 489\end{array}$


SURROUTINE TERM (XOUT, HANMAT, INT, ITP, TEND)

C * TERM CREATES II 700 OUTPUT +ORMAT

IMPLICIT DOUBLE $(A-G, P-Z)$

FARAMETER (NNT $=10),(N N=2 \$ N N I),(N I T=25),(N B K=10),(N N P=N N T+1)$

COMMON /BASISI, I,IBR,NSKIP

CIMENSION XOUT (NNT, G. NIT), HANMAT (NNP, 4$)$, TENO (NIT)

CO $20 \quad J=1$, INTI ITP

PTIME $=$ TEND $(J)$

PRINT 30, HTIME

FRINT 40

CO $10 \quad J K=1$, I

FRINT 50, HANMAT $(J K, 1),(X O U !(J K, J T, J), J T=1,5,2)$

10 CONTINUE

20 CONTINUE

FETURN

LEAF

LEAF

PARAM?

PARAMZ

PARAMZ

LEAF

LEAF

LEAF

LEAF

LEAF

LEAF

LEAF

LEAF

LEAF

LEAF

C

30 FORMAT $1 / 1,2 \times$, $I S S I O N$ PRODUCT INVENTORY A $\#, F 7.2, *$ HOURS IN ATOMS $1 \%, 11$

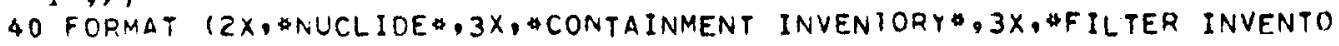
$1 F Y * 3 X_{0}$ INTELRATED RELEASE*,/1

50 FORMAT $(2 x, A 7,7 x, 012,5,10 x, \cup 12,5,8 x, 012.5)$

END

SUBROUTINE PRMAT $(A \cdot I T)$

C \$ FRMAT PRINIS THE A MATRIX

IMPLICIT DCUULE (A-G,P-Z)

FAPANETER (NNT=10), (NN=2*NN!), (NIT=25), (NBK=10), (NNP=NNT+1)

COMMCN /BASISI/ I, IBR,NSKIP

CIMENSION A (NN.NN)

$I P=I+1$

$I 2=I * 2$

$A=1$

FRINT $50.1 T$

FRINT $60, N$

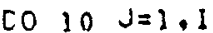

10 FRINT $70,(A, J \cdot J J), J J=[P, I 2)$

$A=N+1$

FRINT $60, \mathrm{~N}$

CO $20 \quad \mathrm{~J}=1.1$

20 FR!NT $70,(A(J \cdot J J), J J=1, I)$

$A=N+1$

FRINT $60 . N$

CO 36 J=ID, IL

30 FRINT $70 \cdot(A(J \cdot J J), J J=1,1)$

$A=N+1$

FRINT $60 . \mathrm{N}$

CO $40 \mathrm{~J}=\mathrm{IP}, \mathrm{IS}$

40 FRINT $70,(A, J \cdot J J), J J=I P \cdot I 2)$

FETURN

C

5O FORMAT $11 H_{1}$.5X. A MATRIX PRINTED BY QUADRANIS FOR TIME INTERVAL*,

LEAF

LEAF

LEAF

LEAF

LEAF

LEAF

LEAF

LEAF

PARAM2

PARAM2

PARAM?

LEAF

LEAF

LEAF

LEAF

LEAF

LEAF

LEAF

LEAF

LEAF

LEAF

LEAF

LEAF

LEAF

LEAF

LEAF

LEAF

LEAF

LEAF

LEAF

LEAF

LEAF

LEAF

$1[3 . / 1$

60 FORMAT $1 / .5 \times$, WUADRANT*, 13,11

70 FORMAT $(2 X, 12(1 \times, 1$ PO 0.03$))$

END

SUBROUTINE SCALAR $(A, S, B)$

C \$CALAR MULTIPLIES A SCALAR IIMES A MATRIX IN COUBLE

IMPLICIT DOUBLE $(A-G, P-Z)$

FARAMETER $(N N T=10),(N N=2 \$ N N !),(N I T=25),(N B K=10),(N N P=N N T+1)$

COMAON /BASISI/ I,IBR,NSKIP

CIMENSION A (NN,NN), B(NN,NN)

$I 2=I * 2$

CO $20 \mathrm{~J}=1.12$

CO $10 \quad J j=1,12$

LEAF
LEAF

LEAF

LEAF

LEAF

LEAF

PARAM2

PARAMZ

PARAMZ

LEAF

LEAF

LEAF

LEAF

485

486

2

4

488

489

490

491

492

493

494

495

496

497

498

499

500

501

502

503

504

505

506

2

3
4

508

509

510

511

512

513

514

515

516

517

518

519

520

521

522

523

524

525

526

527

528

529

530

531

532

533

534

535

536

2

3
4

538

539

540

541 
$E(\lambda \cdot J)=S \# A(\lambda \cdot J])$

LEAF

10 CONTINUE

20 CONTINUE

FETURN

END

SUBROUTINE MULTI $(A, B, C)$

C * NULTI MULTIPLIES TWO MATRICES IN DOUBLE

IMPLICIT DOUBLE $(A-G, P-Z)$

FARAMETER $(N N T=1)),(N N=2 * N N !),(N I T=25),(N B K=10),(N N P=N N T+1)$

COMMON /RASISI/ I, IBR,NSKIP

CINENSION A(NN,NN), B(NN,NN), C(NN,NN)

$12=1 * 2$

Co $20 \quad K=1.12$

CO $20 K K=1.12$

$\triangle M=0.000$

CO $10 \quad J=1,12$

$10 A M=A M+\bar{A}(K, J) \otimes B(J, K K)$

$20(K, K K)=A M$

FETURN

END

SUBROUTINE EQUAL $(A, B)$

C * EQUAL SETS a MATRIX EQUAL TU a MATRIX IN DOUBLE

IMPLICIT DCUELE $(A-G, P-Z)$

FARAMETER $(N N T=10),(N N=2 * N N 1),(N I T=25),(N B K=10),(N N P=N N T+1)$

COMMON /BASISI/ I, IRR,NSKIP

CIMENSION A(NN,NN), 8 (NN,NN)

$12=1 * 2$

10 $30 \quad k=1,12$

ro $10 \quad K K=1.12$

$H(K, K K)=A(K, K K)$

10 CONTINUE

20 CONTINUE

FETURN

ENO

SUBROUTINE MUMUL $(A, B, C)$

$C$ AVMUL DOES PHODUCT OF MATHIX AND VECTOR

IMPLICIT DUUSLE (A-G,P-Z)

FARAMETER (NNT $=13),(N N=2 * N N !),(N I T=25),(N B H=10),(N N P=N N T+1)$ COMMON /BASISI/ I, IBR,NSKIP

CIMENSION A (NN.NN), $B(N N), \subseteq(N N)$

IE $=I * 2$

CO $20 K I=1,12$

$\Delta x_{i}=0.000$

CO $10 X J=1,12$

$10 \Delta M=A M+A(K I, K J) * B(K J)$

$20 C(K I)=A M$

FETURN

END

SUBROUTINE VADD $(A, B, C)$

$C$ * VADO DJES VECTOR ADDITION

IMPLICIT DOUHLE (A-G,P- $Z$ )

FARAMETER $(N N T=1 j),(N N=2 * N N 1),(N 1 T=25),(N B R=10),(N N P=N N T+1)$

COMMON /BASISI, I, IBR,NSKIP

CIMENSION A(NN), B(NN), C(NN)

$12=1 * 2$

CO $10 \mathrm{KI}=1 \cdot I \mathrm{IC}$

$C(K I)=A(K I)+B(K I)$

10 CONTINUE

GETURN

END

LEAF

LEAF

LEAF

543

544

545

LEAF

546

LEAF

547

PARAMZ

PARAMZ

PARAMZ

LEAF

LEAF.

LEAF

LEAF

LEAF

LEAF

LEAF

LEAF

LEAF

LEAF

LEAF

LEAF

PARAM2

PARAMZ

PAPAIIZ

LEAF

LEAF

LEAF

LEAF

LEAF

LEAF

LEAF

LEAF

LEAF

LEAF

LEAF

PARAMZ

PARAMZ

PARAMZ

LEAF

LEAF

LEAF

LEAF

LEAF

LEAF

LEAF

LEAF

LEAF

LEAF

LEAF

PARAMZ

PARAMZ

PARAMZ

LEAF

LEAF

LEAF

LEAF

LEAF

LEAF

LEAF 